\title{
La dette sacrificielle du meurtrier dans les sociétés des bassins des Volta, d'après quelques récits d'administrateurs-ethnologues
}

The murderer's sacrificial debt in societies in the Volta Basin: Following up on the accounts left by colonial officials-ethnologists "

\section{Michel Cartry}

\section{(QpenEdition Journals}

Édition électronique

URL : http://journals.openedition.org/span/1538

DOI : $10.4000 /$ span. 1538

ISSN : 2268-1558

\section{Éditeur}

École pratique des hautes études. Sciences humaines

\section{Édition imprimée}

Date de publication : 1 août 1996

Pagination : 251-304

ISSN : 0294-7080

\section{Référence électronique}

Michel Cartry, "La dette sacrificielle du meurtrier dans les sociétés des bassins des Volta, d'après quelques récits d'administrateurs-ethnologues », Systèmes de pensée en Afrique noire [En ligne], 14 | 1996, mis en ligne le 23 avril 2014, consulté le 09 octobre 2020. URL : http://journals.openedition.org/ span/1538; DOI : https://doi.org/10.4000/span.1538 


\section{LA DETTE SACRIFICIELLE DU MEURTRIER \\ dans les sociétés des bassins des Volta, d'après quelques récits d'administrateurs-ethnologues ${ }^{I}$}

par

Michel Cartry

Au cours de l'année 1931, alors qu'il menait ses enquêtes de terrain chez les Nuer de l'Ouest, Evans-Pritchard se trouva un jour dans un village où régnait un climat de tension et d'inquiétude, et cela, à la suite d'une rumeur persistante autour d'une histoire de meurtre. L'affaire était encore sur toutes les bouches. Un homme venait de mourir et l'entourage du défunt avait fini par se persuader que ce qui avait provoqué la mort de leur parent, c'était la blessure qu'il avait reçue autrefois lors d'une rixe qui l'avait opposé a un homme d'un clan voisin et où il s'était fait agresser à coups de harpons de pêche.

1 Les entretiens que nous avons cus avec divers collègues autour des questions abordées dans cet article ont été pour nous très enrichissants. Pour leurs remarques et leurs suggestions, nous remercions en particulier Hélène Clastres, Marcel Detienne, Michèle Ducornet, Stéphan Dugast, Jean-Louis Durand, et Danouta Liberski-Bagnoud. Certains des faits ethnographiques dont nous faisons état à propos des Lobi nous ont été obligeamment communiqués par Daniela Bognolo et Jeanne-Marie Kambou. Nous remercions également Michel Izard qui nous a communiqué certains textes sur les Mosi ainsi que Marie Raynal pour ses très précieuses fiches de lecture.

Destins de meurtriers

Systèmes de pensée en Afrique noire, 14, 1996 
Dans ce climat de suspicion, l'homme qui avait porté le coup devint bientôt l'objet d'une accusation d'homicide et le risque était grand que les parents du mort ne cherchent à se venger. Pour tenter d'empêcher que ne se produise un conflit armé entre le clan de la victime et celui de l'homicide, des Anciens, partisans de la conciliation, firent appel aux services du "chef à peau de léopard». Au moment où EvansPritchard prit contact avec le village où se passèrent ces événements, les négociations avaient comınencé et on avait déjà arrêté le jour où aurait lieu la cérémonie prévue en pareil cas, cérémonie dont le « chef à peau de léopard», de par son statut, allait devoir être l'officiant principal. Il s'agissait tout à la fois de "purifier" l'homme que l'opinion unanime avait désigné comme l'auteur du coup mortel (une purification pensée dans les termes d'une sorte de « nettoyage du sang répandu ") et d'amener les parties en conflit à accepter le principe d'une conciliation, et cela par un engagement solennel pris devant les dieux de renoncer à la vengeance de sang. Evans-Pritchard fut autorisé à assister à cette cérémonie et pendant plusieurs heures il put observer tout à loisir le déroulement des rites oraux et manuels qui y furent accomplis (Evans-Pritchard, 1940 : 107-111).

Evans-Pritchard n'a sans doute jamais perdu de vue cette observation de référence. En divers textes, il revient sur les questions que soulève l'état d'impureté du meurtrier nuer, et en chacun de ces textes, il arrive à faire passer le vif souvenir des choses vues et entendues lors de la cérémonie dont il a été témoin. A quelques exceptions près, on ne retrouve guère une telle densité d'informations dans les textes que d'autres africanistes, à la même époque, ou dans celles qui l'ont suivie, ont publié sur le même sujet. Quel que soit l'intérêt que présente tel ou tel de ces textes, y manquent, en effet, ces mille détails qui permettent au lecteur d'un travail ethnographique de reconnaître si les informations données sur telle ou telle pratique rituelle s'appuie, ou non, sur une connaissance directe. A partir des années 1930, on entre dans une période de l'histoire des sociétés africaines où les occasions d'observer des rituels liés au meurtre, et à la guerre, se font de plus en plus rares et l'on comprend aisément que les enquêtes menées sur le sujet prennent alors la forme d'une recherche par questionnaires. De tels rituels, quand ils n'ont pas purement et simplement cessé d'exister, ne se 
maintiennent plus que dans une sorte de clandestinité. Avec l'installation d'un nouveau système pénal et de nouvelles formes de normativité pour les affaires dites "criminelles ", ce qui se modifie, c'est aussi la mémoire des idées dont ces rituels étaient l'expression. On pourrait dire que lorsque l'anthropologie professionnelle commença à s'intéresser de façon méthodique aux systèmes rituels africains (autour des années 1950), elle n'avait déjà plus les moyens de se représenter concrètement la façon dont fonctionnait cet aspect de la pratique rituelle $e^{2}$. Bien des anthropologues en délaissèrent l'étude, préférant étudier des rituels encore bien vivants plutôt que de se lancer dans des reconstitutions hasardeuses de pratiques symboliques d'autrefois dont les informateurs ne gardaient que de vagues souvenirs. On peut bien sûr citer quelques textes de cette période qui, sans avoir la qualité de ceux qu'on doit à la plume d'Evans-Pritchard, nous apportent des renseignements substantiels sur la façon dont se déroulait en telle ou telle ethnie le traitement rituel d'un homicide. Des textes de ce genre sont peu nombreux et, s'il n'y avait pas d'autres pieces à verser au dossier, il serait sans doute inutile de relancer l'enquête. Le dossier, heureusement, peut être complété par tout un ensemble de documents d'époque plus ancienne et notamment par ceux qu'on peut trouver dans les grands inventaires ethnologiques établis au début de ce siècle. A cette époque, le colonisateur qui cherchait à mettre en place des juridictions indigènes avait besoin de renseignements sur les coutumes indigènes en matière de réglementation des délits et des crimes et c'est pour répondre à cette attente - de façon plus ou moins directe - que des fonctionnaires de la colonie, parfois aussi des missionnaires s'intéressant à l'ethnographie, ont rassemblé toute une série de données

2 C'est un constat de ce genre qu'est amené à faire Paul Bohannan. Il note qu'à l'époque (1949-1953) où il entreprit ses recherches sur la question de l'homicide et du suicide chez les Tiv du Nigéria, l'appareil juridique mis en place par le colonisateur avait déjà pris en main tout ce qui dans les institutions tiv avait vocation à traiter les * crimes majeurs *. Il ajoute qu'en pays tiv les croyances et les coutumes liées au meurtre ont fait l'objet d'une sorte de * re-institutionalization along British lines *, et qu'en raison même de ce fait, il y a beaucoup d'incertitudes sur les * legal processes which ensue after a homicide * (Bohannan, $1960: 31$ ). 
autour de la question d'un droit criminel indigène où se trouvent souvent d'excellentes informations sur des rituels liés au meurtrier ${ }^{3}$.

Mais s'il est une région d'Afrique où des observations de terrain faites dans les premières décennies de ce siècle, le plus souvent dans le cadre d'enquêtes officielles, ont donné lieu à des textes où la question du meurtre et de ses rites est traitée comme un thème majeur, c'est bien la région des bassins des Volta et plus précisément celle qui correspond au territoire qu'occupent des populations appartenant à l'ensemble linguistique dit « voltaïque ${ }^{4}$. Nombreux sont sans doute les africanistes qui gardent en mémoire le récit saisissant que dans ses Tribus du Rameau Lobi (1931), H. Labouret ${ }^{5}$ nous donne de la longue série d'épreuves rituelles auxquelles on soumet les meurtriers lobi ou birifor pour les protéger - et protéger leur entourage - des effets du khele du meurtre. A ces textes de Labouret, l'on ne peut manquer d'associer ceux de R.S. Rattray, l'un des premiers et des plus profonds connaisseurs des sociétés "voltaïques ». Les pages que l'auteur ${ }^{6}$ de The Tribes of the Ashanti Hinterland (1932) a écrites sur la façon dont certaines tribus du Nord du Ghana prenaient en charge à des fins de purification certaines catégories de tueurs, non pas seulement des

${ }^{3}$ Comme exemples de travaux connus, riches en informations sur un pareil sujet, on peut citer les ouvrages de R.P.J. Roscoe (en particulier 1915 et 1924) et de H. Junod (1912-1913).

4 Il est usage de mettre entre guillemets le terme voltaïque quand on entend désigner par ce terme non les habitants de la Haute-Volta (l'actuel Burkina Faso), mais les populations qui font partie de cette aire culturelle.

5 Après avoir administré la subdivision de Diebougou (1912-1914), peuplée de Birifor et de Dian, H. Labouret fut commandant du Cercle de Gaoua (1914-1920 et 1921-1924), où sont implantées toute une série de populations (dont celle des Lobi), que l'administrateur voulut classer dans l'ensemble dit * Tribus du Rameau lobi *. Plus de 10 années de séjour lui permirent d'apprendre plusieurs langues et de réunir des matériaux ethnographiques d'une grande richesse.

${ }^{6}$ Lorsqu'en 1921 , le capitaine R. S. Rattray commença ses enquêtes linguistiques et ethnologiques au nord du Ghana (ancienne Gold Coast), dans ce qu'on appelait alors l'arrière pays ashanti, il avait déjà effectué un immense travail de recherche sur les Ashanti eux-mêmes, travail dont il publia les résultats dans une série d'ouvrages qu'on peut aujourd'hui considérer comme des classiques. Fonctionnaire du Colonial Office, la qualité de ses travaux lui valut d'être affecté à un poste d'anthropologue du gouvernement. 
tueurs au retour d'expéditions guerrières, mais aussi différentes sortes de meurtriers, sont parmi les plus denses qu'on ait jamais produites sur un pareil sujet.

Ce que nous allons tenter d'articuler ici sur la dimension sacrificielle des pratiques de réparation dont sont l'objet les personnes, les choses, et les lieux affectés par le sang du meurtre s'inscrit dans le prolongement direct des questions que se sont posées ces deux auteurs dans les ouvrages mentionnés. Tout au long de notre enquête, nous nous laisserons guider par leurs intuitions. Dans les sociétés * voltaïques ", les procédures rituelles auxquelles on a recours pour traiter des problèmes déclenchés par la mise à mort d'un homme (ici un risque de pollution pour toute une communauté, ailleurs, un mal mystérieux, souvent contagieux, affectant le corps du meurtrier) se présentent souvent sous l'aspect d'un dosage complexe d'actes de nature variée comprenant des bains, des fumigations, des pratiques magiques, des absorptions de breuvages, des confections d'amulettes ou de fétiches, des rites mimétiques, des jeûnes, etc. Presque toujours, on y trouve aussi des opérations de type sacrificiel, certaines d'entre elles présentant un certain degré d'atypisme, soit qu'on y immole un animal « mystiquement » dangereux, soit qu'on y répande le sang sur le corps même des sacrifiants, soit qu'on y traite la part consommable des victimes offertes aux dieux avec une crainte particulière. Ce que Rattray et Labouret nous ont apporté sur cet aspect de l'activité rituelle, c'est bien davantage qu'une documentation de " première main ", riche et précise. On leur doit notamment une série de repérages sur les schemes d'action suivis en de tels sacrifices, repérages qui laissent parfois entrevoir la façon dont on se représente les effets de souillure produits par le sang du meurtre et ce qui dans cette souillure risque de porter atteinte aux rapports fragiles d'équilibre qu'une communauté entretient avec ses dieux. Les pages où Labouret décrit les séquences sacrificielles qui prennent place dans la «cure " d'un meurtrier lobi ou birifor projettent un vif éclairage sur la façon dont le meurtrier est atteint en son corps par les puissances du meurtre qu'il a lui-même suscitées ainsi que sur les procédés dont use le rite pour traiter ce corps avec le sang du sacrifice (par exemple, ces pages présentant les opérations du rite traitant l'arme du meurtrier ainsi que la 
main qui a tenu cette arme comme une sorte d'autel vivant). Au fil des nombreux récits que nous livre Rattray sur le déroulement de cures semblables, l'on découvre des modèles de rites sacrificiels d'un très grand intérêt, modèles où l'on voit parfois se refléter ce qui pour une société donnée caractériserait en propre le destin d'un meurtrier.

Mais chez les "Voltaïques ", il est un autre aspect de la pratique sacrificielle qui dans l'optique qui est la nôtre - celle d'une recherche visant à saisir au travers d'opérations sacrificielles certaines formes de réponse rituelle qu'apporte une société à l'acte meurtrier - n'est pas moins intéressante à étudier que celui que nous venons d'évoquer à propos des rituels de purification centrés sur le meurtrier. En ces sociétés, il arrive parfois que l'acte de verser le sang humain soit "sanctionné » par un rite sacrificiel dont la prise en charge devra être assurée par le «maître de la Terre". A ce rituel, devront parfois participer le meurtrier ou certains de ses proches, mais ce n'est pas vraiment la question de leur impureté qui est au centre du rite. On ne peut définir d'un mot ce qu'est l'objet propre de ce type de sacrifice, mais on peut déjà dire que la forme de détérioration consécutive à l'événement du meurtre, qui le rend nécessaire, met en jeu une problématique de la souillure qui n'est pas de même nature que celle à laquelle sont confrontés les agents du rite spécialement chargés de diriger les " cures » de meurtrier. Il y a là deux domaines de l'activité sacrificielle qui assurément " $s$ 'interconnectent " mais qui relèvent de logiques différentes. Ce n'est pas, on s'en doute, à la suite de n'importe quel meurtre que le maître de la Terre est appelé à intervenir pour réparer les effets de souillure qu'on associe parfois au sang du meurtre. Il n'intervient que dans les cas où l'agent du meurtre, de par son acte, a violé l'un de ces interdits fondamentaux qu'on appelle en ces sociétés des «tabous de la Terre ». Qu'est-ce qu'un tabou de la Terre ? Qu'est-ce qui lie l'interdit du meurtre à d'autres sortes d'interdits du type tabou de la Terre ? Ce que Labouret et Rattray ont cherché à construire autour de ce thème permet un certain repérage de ce qui est en jeu dans les réparations sacrificielles qu'entraîne la transgression d'un tabou de cette sorte. Avant d'étudier sur la base d'un exemple emprunté à Rattray la dimension sacrificielle des rituels de purification centrés sur le meurtrier, il convient de restituer la démar- 
che suivie par les deux auteurs pour aborder cet autre aspect de l'activité sacrificielle liée au meurtre.

\section{Le sang du meurtre, et les tabous de la terre}

Bien que Labouret et Rattray aient construit des ethnographies dont les orientations théoriques sont parfois divergentes, on ne peut manquer de relever un interêt commun pour tout un ensemble de questions touchant les fondements religieux du droit. L'attention extrême que l'un comme l'autre ont porté à cette figure d'autorité qu'est le maître de la Terre, si présente dans les sociétés qu'ils étudiaient, témoigne de cet intérêt ${ }^{7}$. Au terme d'une étude sur le sens ancien du mot rex dans certaines langues indo-européennes, Benveniste avait réussi à isoler un ensemble de notions liées à l'histoire de ce mot, d'où il ressortait que le rex en sa figure archaïque était un personnage dont la mission n'était pas de commander mais de " tracer la ligne ",

7 Les formes d'organisation politique qu'ont élaborées les sociétés de I'aire culturelle * voltaïque * sont fort variables (sociétés sans chefferies dites acéphales; sociétés où des chefs politiques sans grand pouvoir et des " prêtres de la Terre * exerçaient ce que Rattray appelait un * dual mandate *; royaumes fortement centralisés dotés d'un appareil complexe de gouvernement), mais il a été maintes fois constaté qu'il n'y en a qu'un petit nombre qui n'ait pas fait une place importante à l'institution des maîtres de la Terre. Cette institution n'est certes pas une caractéristique des systèmes sociaux et religieux des sociétés * voltaïques *, mais l'on doit toutefois reconnaître que dans les sociétés de cet ensemble, elle s'exprime en des formes rituelles si semblables qu'on ne peut s'empêcher de penser à des élaborations particulières faites sur la base d'un héritage culturel commun. Ceci une fois posé, l'existence d'invariants nombreux ne doit pas faire perdre de vue les différences. Chez les Lobi, le "maître de la Terre * n'a pas à partager ses prérogatives avec un chef issu d'un clan aristocratique. Chez les Nankanse du Nord du Ghana étudiés par Rattray, il existe des situations où le maître de la Terre et le chef sont amenés à coopérer dans l'exécution de tâches rituelles. Dans cet article, nous aurons souvent l'occasion de passer en revue des exemples divers d'interventions du maître de la Terre observés en diverses sociétés "voltaïques *. Compte tenu de la nature de notre propos, nous mettrons surtout l'accent sur les ressemblances sans trop tenir compte des particularismes des ethnies où nous avons puisé nos exemples. Le lecteur comprendra que si nous avions voulu entreprendre une étude comparative des fonctions du maître de la Terre dans les régions des Bassins des Volta, nous aurions choisi un autre mode d'exposition. 
de déterminer ce qui est, au sens propre, « droit » (Benveniste, 1969). On serait tenté d'appliquer cette formule au personnage du maître de la Terre. Privé lui aussi de toute fonction de commandement, cette sorte de « roi prêtre » (ainsi l'appelait Rattray) n'a pas d'autre pouvoir que celui de dire « ce qui est droit " dans certaines situations de danger où se trouvent placés les gens d'une « même terre » après la rupture de certains interdits fondamentaux. De par le statut que lui assigne le mythe d'être un enfant « né de la Terre ", ce personnage est comme le témoin d'un monde de l'origine où la Terre et le Ciel n'étaient pas encore séparés ${ }^{8}$ et c'est bien cette origine qui donne autorité à son dire et à ses gestes en ces moments de la vie du village où les circonstances l'obligent à réénoncer ce que sont ces interdits fondamentaux que depuis Rattray on a coutume d'appeler " les tabous de la Terre ".

Que l'on ouvre n'importe quel ouvrage d'ethnographie consacré à ces populations « voltaïques " (non seulement ceux de Labouret et de Rattray, mais aussi ceux qu'on doit à des auteurs plus récents) ${ }^{9}$, l'on constate que, d'une population à l'autre, la liste qui est donnée de ces tabous de la Terre est à peu de choses près toujours la même. Dans

8 Pour désigner ce que ce que nous appellerons tantôt la Terre, tantôt le sol, tantôt le pays, la plupart des populations qui appartiennent à l'aire linguistique * voltaïque * utilisent des termes qui sont forgés sur le même radical et dont les champs sémantiques se recouvrent presque complètement. Ces termes correspondent à une notion complexe qu'il est difficile de rendre dans nos langues car si le contexte d'utilisation permet parfois de faire un choix entre plusieurs traductions possibles, il arrive très souvent qu'on puisse hésiter entre l'un ou l'autre des sens suivants : celui de pays ou de * land *, celui de village ou de localité, celui d'un lieu * sacré " déterminé du territoire villageois, celui d'une étendue de terre conçue comme le domaine sur lequel s'étend la juridiction d'un seul * mâ̂tre de la Terre *, celui, enfin, de Terre dans son aspect de puissance mystique unique et universelle dont tous les hommes dépendent.

${ }^{9}$ La littérature ethnographique consacrée à l'institution des maîtres de la Terre des populations * voltaïques * qui a été publiée jusqu'à ce jour est très riche. Rappelons que les grandes monographies de Meyer Fortes (1945 et 1949) et de Jack Goody (1956 et 1962) portent sur des populations de cette aire culturelle, les Tallensi du Nord du Ghana, d'une part, les Lo-Dagaa, de cette mêrne zone, d'autre part. Ces deux auteurs rendent plusieurs fois hommage à Rattray qui avait lui-même étudié ces deux populations. Ces quinze dernières années des recherches intensives de terrain menées chez les Lobi se sont multipliées et ont donné lieu à des travaux de qualité ( $c f$. ouvrages cités en référence). 
cette liste, on trouve d'abord un groupe d'interdits dont l'objet n'est pas le meurtre en tant que tel mais le meurtre sanglant ainsi qu'une série d'actes d'agression corporelle n'ayant pas entraîné la mort mais ayant provoqué un écoulement du sang sur le sol (ainsi le fait d'avoir infligé une blessure lors d'une rixe où se sont affrontés des gens d'une même terre). Sont inclus dans cet ensemble certaines formes de suicide (se donner la mort avec une pointe de flèche) ainsi que des actes où la blessure reçue semble accidentelle mais qui devient rituellement signifiante en raison des circonstances. Des faits recueillis chez les Kasena par D. Liberski lors d'enquêtes ethnographiques récentes font entrevoir la façon dont s'opère cette transformation : un cultivateur kasena qui en travaillant son champ s'était blessé accidentellement se trouva bientôt en situation d'avoir à payer au maître de la Terre une sorte d'amende sacrificielle, car selon les dires de son entourage, il avait répandu son sang en une période de l'année jugée particulièrement dangereuse. Ce dernier exemple montre que « l'intention n'est nullement requise pour faire d'un acte une faute rituelle (Mauss, 1969b: 694) en rapport avec les tabous de la Terre. D'autres exemples tendent à montrer qu'entre les tabous de la Terre liés au sang et ces autres sortes de tabous qui concernent spécifiquement le sang féminin, il y a parfois de réelles connexions : il est rapporté que lorsqu'une femme accouche hors du village en un lieu dit « de brousse », elle est tenue comptable du sang répandu sur le sol et cela comme si cette forme d'écoulement de sang la plaçait - et plaçait aussi son mari - dans la même situation rituelle que ceux qui ont enfreint un tabou de la Terre en versant le sang par un acte de violence.

Mais dans ce groupe des tabous de la Terre, le sang n'est pas toujours en cause ${ }^{10}$. S'approprier pour son usage personnel ce qu'on

10 Comme autre exemple de tabous non liés à une affaire de sang, on peut signaler celui qui fait défense d'avoir des rapports sexuels en des lieux non habités, jugés * incultes * ou * sauvages *. Chez les Kusase (nord du Ghana), l'homme qui surprend le couple doit collecter un tas de feuilles et de branches et l'amonceler au lieu du coït. A chaque fois qu'un passant apercevra le lieu ainsi marqué, il devra rajouter des rameaux de branches et de feuilles. Chez les Kasena, le responsable rituel de la portion de terre où se trouve le lieu de la transgression devra y faire un sacrifice pour * ramasser la saleté * (Liberski, infra). 
a trouvé par hasard sur un territoire comme "épaves " de toutes sortes (animaux égarés, biens perdus non réclamés par leurs propriétaires, choses de provenance inconnue) est un acte qui fait aussi partie du même type d'infraction. Ces choses reviennent de droit à la puissance Terre et leur seul dépositaire légitime est le maître de la Terre. Le découvreur devra donc sans tarder les lui confier pour qu'il les restitue à la Terre, ce qu'il fera soit en les lui consacrant, soit en s'en servant au titre de paiements sacrificiels.

Il arrive que certaines ethnies * voltaïques » intègrent encore dans ce même ensemble non pas des actes mais des abstentions d'actes ou plutôt ce qu'on pourrait appeler des manquements - ou des omissions - rituelles. Comme la plupart des sociétés africaines, les sociétés "voltaïques » ont fixé jusqu'au moindre détail les coutumes funéraires et en particulier celles qu'on doit suivre dans les heures qui suivent le décès quand il s'agit de prendre une décision quant au type de sépulture qu'on doit donner au parent qu'on vient de perdre. Pour faire face a ce genre de situation, la famille qui conduit le deuil devra faire appel au concours de personnes qui exercent - et parfois à titre héréditaire - la fonction de fossoyeurs, mais le choix d'une sépulture fait intervenir un nombre si important de criteres qu'il arrive parfois que, sans le savoir, elle fasse un mauvais choix, se mettant ainsi en situation d'infraction rituelle. Un certain nombre de ces infractions risquent de porter atteinte a Terre en tant que puissance dont la sphère de souveraineté s'étend aussi au monde des morts et des ancêtres et qui, à l'échelle d'un village ou d'un territoire déterminé, n'est censé accepter en son sein que certaines catégories de mort. En tant qu' « enfant de la terre ", le maître de la Terre est l'interprète qualifié des discriminations que la puissance Terre ne cesse d'opérer entre les humains du point de vue de leurs "destins de sépulture " ${ }^{11}$. A ce titre, il a bien un droit de regard sur la façon dont les gens qui dépendent de sa terre prennent en compte au moment voulu ces destins de sépulture.

Cette fonction de surveillance, il l'exerce de façon discrète et s'il arrive qu'en certaines situations, il soit amené à intervenir pour régler

11 Nous empruntons cette expression à Michel Guibal (1984). 
un problème de sépulture, il le fait sur la base de critères qui lui sont propres et pour des tâches spécifiques qui n'interferent qu'en partie avec celles qui relevent de la fonction de fossoyeur. Rattray nous indique que chez les Dagaba du Nord du Ghana, les tendagena (" maîtres de la Terre »), après constat de certaines infractions commises par la famille en deuil, peuvent suspendre pour un temps le déroulement des funérailles (Rattray, 1932: 420). A propos des Kusase, ce même auteur cite le propos que lui livra un maître de la Terre pour justifier son droit de regard sur la question des sépultures : pour certains types de morts (ainsi ceux qui laissent une descendance), soulignait l'informateur de Rattray, il ne faut pas attendre trop longtemps pour procéder à la mise en terre ; si l'on attend que le corps pourrisse (rotten) avant de l'enterrer, ajoutait-il, alors on risque de polluer (spoil) la terre (Rattray, 1932: 391).

La liste des tabous de la terre que nous venons de donner est loin d'être complète et l'on pourrait encore faire état de situations d'infraction rituelle encore plus insolites que celles dont nous venons de parler, comme par exemple celles où peuvent se trouver placés des villageois que le hasard de leurs pérégrinations amènent à découvrir sur une terre de brousse le cadavre d'un inconnu.

Quand, dans tel ou tel village, viennent à se produire des événements comme ceux que nous venons de décrire, le maître de la Terre en est, en principe, aussitôt informé. On n'a pas beaucoup de renseignements sur la façon dont ce personnage, une fois saisi de l'affaire, doit se comporter pour arriver à déterminer si l'événement qui s'est produit met en cause un tabou de la Terre, mais on peut supposer qu'il ne rend pas son arrêt à la légère ${ }^{12}$. La chose une fois " jugée ", il doit alors faire connaître aux parties concernées ce qu'elles auront à faire dans le rite de réparation qui s'impose en la circonstance. Sa

12 Déceler dans l'événement la transgression d'un tabou de la terre et deviner les sanctions auxquelles le village est exposé en conséquence de cette transgression (risque de sécheresse, d'épidémie) sont les deux aspects complémentaires de sa fonction d'interprète. Il arrive aussi assez souvent que le maître de la Terre, remontant le cours du temps, soit amené à rechercher la cause d'un malheur déjà arrivé mais encore inexpliqué dans une transgression passée non traitée rituellement. A propos des LoDagaa, J. Goody analyse longuement une situation de ce type (Goody, 1962). 
fonction essentielle, nous l'avons vu, n'est pas de punir (certaines de ses interventions par le biais d'imprécations, de malédictions, ou d'ordalies de toutes sortes, comportent toutefois un aspect punitif), mais d'obliger les parties en cause à venir présenter aux dieux ce qui leur est réclamé au titre de paiements sacrificiels.

Bien que Labouret fasse maintes fois allusion aux paiements sacrificiels dus à la Terre en des situations de ce type, il ne fournit que peu de détails sur la conduite des rites eux-mêmes. Rattray est sur ce point plus précis. On trouve toujours chez cet auteur, même en ses compte-rendus les plus brefs, quelques informations sur les gestes, les lieux et les paroles qui permettent d'entrevoir la finalité de tels sacrifices.

Si l'on prête attention à ce que dit l'officiant dans sa prière en ce moment du rite où il expose aux dieux la raison de sa présence et le sens des gestes qu'il est en train d'accomplir, l'on remarque qu'il puise ses métaphores dans une série de verbes qui dans leur emploi courant signifient « ramasser », " nettoyer », "balayer ", " éteindre ", " enfouir ». "Ils sont venus pour ramasser le sang ». Ou encore : «Ils sont présents en ce lieu pour ramasser la saleté ". De telles expressions abondent dans les comptes rendus de Rattray, expressions que, lors de recherches récentes, D. Liberski a retrouvées chez les Kasena. Ce chercheur a pu noter que c'est bien en ces termes que s'exprime le prêtre de la Terre kasena lors de rites de réparation effectués pour le compte de gens de sa terre tenus comptables, pour les uns, d'avoir versé le sang humain (première formule), pour les autres, de s'être laissés prendre dans l'espace de contagion d'un cadavre laissé sans sépulture (deuxième formule). Les conceptions de la souillure que les sociétés “ voltaïques " ont élaborées sont encore très mal connues et il y a lieu d'être prudent quand on tente d'interpréter en termes de souillure la nature de l'atteinte qui est faite au territoire. Que la transgression ait pour effet de " gâter " ou de " salir » ou de «polluer " ou "d'incendier " le lieu même où elle s'est produite, c'est bien en tout cas ce que font apparaître certaines formules du rite.

Lorsque Rattray et Labouret, en tel ou tel chapitre de leur ouvrage, abordent la question des tabous de la Terre, il leur arrive parfois d'introduire dans leurs comptes rendus une série de réflexions 
où après avoir dit leur étonnement par rapport à l'étrangeté des croyances qu'ils exposent, ils en viennent à s'interroger sur la place qu'occupe la notion de délit et de crime en des systèmes sociaux où le juridique n'est pas séparable du religieux. Ce qui avait tout particulièrement intrigué ces deux auteurs dans les procédures de réparation que conduit le maître de la Terre, c'est le fait qu'elles plaçaient sur le même plan deux catégories bien distinctes de " transgresseurs ": l'une faite de personnes qui, à l'évidence, avaient bien commis un délit grave (certains meurtriers), l'autre, faite de gens, dont on ne voyait pas d'emblee, du moins dans les termes du droit occidental, quelle sorte de faute ils avaient au juste à réparer. Labouret et Rattray connaissaient sans doute assez bien les controverses anthropologiques de leur époque sur la question des origines du droit criminel ${ }^{13}$ et l'on pourrait montrer que le souci qu'ils avaient, l'un et l'autre, d'apporter leurs contributions personnelles à cette sorte de débat a été pour eux un puissant stimulant dans l'attention extrême qu'ils ont porté à l'institution des tabous de la Terre ainsi qu'au système des sanctions qui lui est lié. Au tournant du siècle, l'idée selon laquelle la catégorie du meurtre dans les sociétés archaïques est entièrement prise dans le moule de ce que Gernet appelait (M. Detienne nous le rappelle ici) " une forme de pensée mythico-religieuse » complètement indifférente aux distinctions qui nous sont devenues familieres entre plusieurs catégories d'homicides, était partagée par un grand nombre de savants, anthropologues ou historiens. Qu'une pareille construction intellectuelle ait fourni à Labouret et Rattray l'étayage sur lequel ils se sont appuyés pour entreprendre sur le terrain un travail de collecte minutieux de données relatives au sang du meurtre, aux tabous de la Terre, et aux réparations sacrificielles faites sous l'égide du maître de la Terre, cela ressort de

${ }^{13}$ Labouret et surtout Rattray qui avaient reçu une formation poussée en anthropologie connaissaient non seulement les travaux classiques consacrés à la notion de Tabou mais aussi les tentatives faites par certains historiens d'appliquer à l'étude des institutions juridiques de l'Antiquité certains concepts ethnologiques. En plusieurs passages des Tribus du Rameau Lobi, on reconnait l'influence directe qu'exerça sur Labouret les opinions qu'avait soutenues Mauss dès 1896 dans son compte-rendu critique de l'ouvrage de M.R. Steinmetz (Mauss, 1969b) 
nombreux passages de leurs ouvrages ${ }^{14}$. Les spéculations autour de la notion de tabou nous paraissent aujourd'hui bien fragiles, mais l'on doit pourtant reconnaître qu'elles ont parfois été à l'origine d'enquêtes de terrain d'une très grande richesse. Sans ces spéculations, Labouret et Rattray n'auraient peut être pas osé imaginer que les tabous de la Terre, en apparence si hétéroclites, s'organisaient en un ensemble cohérent. S'ils ne sont pas parvenus à dégager toutes les articulations d'un tel système, ils nous ont fait entrevoir un aspect de la question de l'interdit du meurtre qui n'avait pas suffisamment retenu l'attention des historiens et des anthropologues. Tous deux ont senti que l'aspect sous lequel le maître de la Terre traitait la question du meurtre dans ses rituels de réparation n'était pas celui que privilégient nos constructions juridiques et pénales, mais ce qui dans l'acte de verser le sang humain sur la terre où l'on vit pourrait avoir pour effet d'y meurtrir tout ce qui est vivant. On peut aussi porter à leur crédit d'avoir su deviner que ce qui surgit comme question liée à la souillure dans les violations des tabous de la Terre ne se limitait pas à des affaires de sang versé. On doit enfin leur attribuer le mérite d'avoir su reconnaître que la procé-

14 De par les fonctions officielles qu'ils exerçaient, Labouret (l'administrateur) et Rattray (l'anthropologue du gouvernement) conduisirent leurs recherches de terrain avec une optique * d'anthropologie appliquée *. Ils ne perdirent jamais de vue l'objectif pratique qui leur était assigné, celui d'avoir à expertiser des situations de dysfonctionnement dans la mise en place des structures administratives de type colonial. S'il est un domaine de la politique coloniale sur les dysfonctionnements duquel ces deux * experts * ne cessèrent de s'interroger, c'est bien celui qui concerne l'administration de la justice. Ayant eu à exercer la fonction de magistrat, Labouret tire parfois la leçon de son expérience. Il note à plusieurs reprises qu'entre ses administrés il existe une sorte de complicité tacite pour ne pas porter certaines affaires - dont des affaires * criminelles * - devant les tribunaux de la colonie. Rattray n'a pas connu cette expérience, mais il porte un jugement critique sur le fait que l'administration coloniale ait cru bon de confier des pouvoir * judiciaires * exorbitants à de petits chefs locaux - souvent imposés de force aux villageois - qui ignorent tout des principes et des règles du droit coutumier ou de l'institution des * maîtres de la Terre *. Qu'une accusation portée contre quelqu'un puisse se retourner contre l'accusateur, devenant ainsi une puissance qui mettrait en péril la vie de ce dernier, et le contraindrait à faire l'acquisition d'un autel de protection, c'est cette croyance qui, selon Rattray, permettrait d'expliquer pourquoi les villageois répugnaient à aller porter une affaire devant les tribunaux et cela surtout quand il s'agit d'une affaire criminelle où le coupable est passible de la peine de mort. 
dure majeure à laquelle les sociétés "voltaïques " ont recours pour effacer la souillure qui pollue la terre - souillure du sang ou autre forme de souillure - est le geste du maître de la Terre répandant sur le sol le sang des victimes sacrificielles.

Les points de convergence que nous venons de signaler entre Labouret et Rattray dans leurs approches respectives tant des violations des tabous de la Terre que des rites de réparation qu'elles impliquent, ne doivent pas toutefois nous faire perdre de vue ce qui constitue l'optique propre de ces auteurs dans la maniere de conduire l'analyse des faits.

Ce qui laisse insatisfait dans le schéma d'ensemble que propose Labouret pour tenter d'expliquer les croyances et les rites lobi liés à la notion de souillure dans le contexte des tabous de la Terre, c'est le fait qu'il semble davantage dérivé d'emprunts divers aux théories anthropologiques de son époque que d'une tentative d'élaboration personnelle des données observées, libre de tout à priori d'Ecole. Labouret a emprunté à Marcel Mauss $(1969 b)$, lecteur de M.R. Steinmetz ${ }^{15}$, l'idée que dans beaucoup de sociétés primitives, ce n'est pas tant le meurtre qui constitue, au sens propre du terme, l'acte criminel, mais le sacrilège. C'est autour de cette notion de sacrilège, telle qu'elle fut réélaborée par Mauss, que tournent la plupart des propositions générales qu'avance Labouret lorsqu'il tente d'expliciter la conception lobi de cette espèce particulière d'infraction religieuse qu'est la violation

15 En 1894, ce grand érudit qu'était Steinmetz avait fait paraître à Leipzig un ouvrage qui sur la base d'un nombre impressionnant de documents ethnographiques se proposait d'établir une théorie nouvelle de la peine en montrant que la peine familiale est la source d'où provient la * vindicte publique des peuples civilisés * et donc aussi, avec celle-ci, les premières ébauches du système pénal. En conclusion de son compte rendu, Mauss, au demeurant très élogieux, reprochait à cet auteur d'avoir cru à tort que les formes premières de la réaction pénale publique dérivent - logiquement et historiquement - des réactions que déclenche le meurtre d'un parent dans les système sociaux qui pratiquent la vengeance de sang. Selon Mauss, Steinmetz serait resté obnubilé par l'idée que dans toutes les sociétés - dans les sociétés primitives comme dans les nôtres - le véritable acte criminel, c'est le meurtre. C'est à cause de ce préjugé, ajoutait Mauss, que l'historien du droit n'aurait pas su reconnaître le caractère pénal des sanctions - réelles ou mystiques - dont sont l'objet, dans les systèmes les plus archaïques du droit, les individus qui ont violé un tabou religieux. 
d'un tabou de la Terre. Dans le prolongement des réflexions de Durkheim sur la peine et la religion, Mauss avait tenté de montrer que dans les sociétés de "pré-droit " (expression de Gernet, nous rappelle ici M. Detienne), il y a une multitude de défenses religieuses qui, à la différence d'autres sortes d'interdits religieux, présentent les traits distinctifs suivants : 1) elles ont un caractère public ; 2) elles sont toujours assorties d'une menace de sanction à caractère mystique pouvant entraîner la mort ou quelque calamité ; 3) elles prévoient pour les transgresseurs des amendes rituelles ou des rites de rachat ou d'expiation. De telles défenses, ajoutait Mauss, ressemblent à celles qu'édictent certaines sociétés historiques par rapport aux actes dits sacrilèges, mais elles sont plus difficiles à identifier car elles mettent en jeu cette logique particuliere que nous a rendu familière la découverte de l'institution religieuse du tabou : quel que soit leur but lointain, elles ont pour objet immédiat une prohibition au sens strict de ce terme, celle d'avoir a éviter certaines formes de contact par rapport a des choses sacrées ou " tabouées ". Certaines formes d'inceste, certains types de profanation, le régicide, certaines formes de sorcellerie, mais aussi certaines sortes d' " injures " au sang, entrent dans la liste de ces crimes religieux, liste dont ne fait pas partie l'homicide en tant que tel.

C'est cette thérie de Mauss qui va fournir à Labouret les concepts dont il a besoin pour forger la grille de lecture qu'il va appliquer aux faits lobi. Chez les Lobi, déclare en substance Labouret, tous les dieux sont subordonnés à la puissance Terre, puissance qui règne sur son domaine à la manière du seigneur des lieux et qui fait respecter sa loi en répandant sur les autres dieux, les choses, les êtres et les lieux qu'elle veut protéger - ou garder purs de tout contact - le khele, sorte de fluide nocif qui d'abord rayonne du corps des dieux, mais qui du fait d'une violation peut atteindre toute personne qui aura franchi la limite qui sépare le monde des choses ordinaires de ce monde protégé, excepté. A chaque fois que Labouret cherchera à rendre compte des effets de souillure contagieuse que provoque la violation d'un tabou de la Terre, c'est à cette théorie généralisée d'une énergie se déplaçant à la manière d'un fluide qu'il se référera. On comprendra aisément que l'utilisation quasi mécanique de cette théorie - une variante de la théorie du mana en sa version maussienne - ait 
pu détourner l'auteur de l'ouvrage Les Tribus du Rameau Lobi de la tâche (à la fois plus modeste et plus difficile) d'expliquer ce qui dans telle ou telle situation de transgression fait effet de souillure. On comprendra aussi qu'une telle theorie n'assigne au meurtre qu'une place marginale dans l'ensemble des transgressions des tabous de la Terre. Chez les Lobi, précisera Labouret, le meurtre n'est traité comme un "crime " que dans les cas où le meurtrier a commis une infraction religieuse en versant le sang ${ }^{16}$. Verser le sang de l'autre dans les limites de l'agglomération que commande le seigneur du domaine est un acte qui expose au khele du sang, substance qui, en pareil cas, sera susceptible de contaminer aussi bien le corps dont elle est sortie que celui qui l'a répandue, mais encore l'instrument du meurtre ou le lieu de la terre où elle a été répandue.

Labouret a écrit de très belles pages non seulement sur les rituels de purification d'un meurtrier ${ }^{17}$, mais aussi sur la question des tabous de la Terre. On ne peut suivre cet auteur dans l'interprétation qu'il a proposée de la conception lobi de la souillure. La distinction qu'il a faite entre crime ordinaire et crime religieux n'est pas très convaincante. Peu crédible est l'idée qu'il n'y aurait pas de place dans le système de pensée des Lobi pour la catégorie générale d'un interdit du meurtre, mais seulement pour l'institution d'un tabou du sang dont celui lié au sang du meurtre ne serait qu'un cas particulier. L'assimila-

${ }^{16}$ Aux pages 377-380 de son ouvrage, Labouret, après une remarque générale sur la notion de crime indique : * ici un crime n'est pas seulement comme chez nous une violation du droit, provoquant une réaction de la société...*. Un peu plus loin, il écrit : * la question se pose de savoir s'il convient de ranger dans la même catégorie (celle de 'crimes religieux') les actes de violence avec effusion de sang. Il semble bien que ces fautes indisposent la Terre...*. Cette position, il la réénoncera quelques lignes plus loin : le meurtrier n'est pas considéré comme coupable par ses parents puisque sa victime est un étranger, * mais on admet qu'il a commis une infraction religieuse en versant le sang, c'est pourquoi j'ai signalé plus haut qu'on pouvait faire entrer le meurtre dans la catégorie des fautes religieuses à racheter et à expier * (Labouret, opus cit : 380).

17 Pierre Bonnafé et Michèle Fiéloux (1984), qui ont effectué de longues enquêtes de terrain chez les Lobi, ont récemment proposé une lecture très intéressante de ces pages de Labouret. Dans une thèse encore inédite, Michèle Cros (1987) a repris l'analyse méthodique de toutes les acceptions de la notion de khele. 
tion du meurtre sanglant à un sacrilège nous paraît, en outre, quelque peu forcée car elle repose sur un ensemble de notions - celles de faute, de rachat et d'expiation - qui, au regard des faits décrits, ne semble guère s'imposer. Des documents que cet auteur lui-même a rassemblés, il ne ressort pas clairement que les rites lobi liés à la réparation du sang versé relèvent d'une logique de l'expiation. Cette notion d'expiation n'a aucune pertinence dans le contexte de cette longue série de rites qui visent à traiter ce mal mystérieux dont sont atteints, sans exception, tous les individus qui ont versé le sang humain. Mais cette notion ne s'impose pas non plus dans les cas où la reparation rituelle prend la forme de sacrifices offerts à la puissance Terre sous la responsabilité de son prêtre ou de l'un de ses representants. On peut bien accepter la métaphore quasi universelle d'un sang sacrificiel qui laverait la souillure du sang du meurtre, mais il n'est peut-être pas nécessaire d'y adjoindre l'imagerie d'une divinité offensée dont on détournerait le courroux sur des victimes expiatoires.

Tout autre est l'horizon intellectuel de Rattray. Cet auteur était lui aussi nourri de lectures anthropologiques sur l'interdit ${ }^{18}$, mais il a eu

${ }^{18}$ Rattray connaissait bien les théories de Frazer tant sur la question du roi divin que sur celle des interdits de type * tabou * et si les portraits qu'il a composés des maîtres de la Terre rencontrés dans les sociétés du Nord Ghana ont une incontestable originalité, ils sont souvent assortis de considérations qui portent la marque des idées qu'avait développées Frazer sur les formes archaïques de la royauté. Comme le roiprêtre des bois sacrés frazériens, le maître de la Terre "voltaïque "apparait sous la plume de Rattray comme un personnage tout à la fois vénéré, effacé, "cloîtré *, entouré de tabous, parfois installé et intronisé de force, pouvant décréter mille tabous mais "sans bras exécutif *, gardien de la Terre mais laissé à l'écart des affaires séculières. Cette filiation une fois rappelé, ce qu'a découvert Rattray sur le rôle sacrificiel spécifique que joue le maître de la Terre * voltaïque " dans les affaires de sang ne doit rien à Frazer. Dans Tabou et les périls de l'âme, Frazer (1951) avait soutenu l'idée que dans un grand nombre de sociétés primitives, les rites de purification auxquels sont soumis les guerriers au retour d'une bataille victorieuse contre l'ennemi ont la même finalité que les mises en quarantaine rituelles dont sont l'objet " ceux qui ont souillé leurs mains du sang d'un membre de la tribu ". Comme Frazer, Rattray a constamment réaffirmé l'idée que dans certaines sociétés - celles où le meurtre n'est pas une catégorie juridique - le fait de verser le sang de l'Autre est toujours perçu, quel que soit le contexte (à la guerre, en une situation de vengeance, en réponse à une impulsion incontrôlée contre un parent ou un voisin), comme un acte qui met en état de danger * mystique * celui qui l'a accompli. C'est le seul point de 
trop le souci de laisser ouvert le champ des questions qu'appelait son ethnographie, trop le souci également de ne pas faire violence aux riches métaphores qu'il savait entendre dans le discours de ses informateurs pour s'être senti obligé d'avoir à situer ses propres découvertes par rapport à des théories déjà connues. A propos de certaines transgressions de tabous de la Terre, Rattray s'exprime parfois en des termes qui tournent également autour de la notion de pêché ou celle "d'acte d'impiété " (viennent parfois sous sa plume des formules " style Labouret », du type « la Terre a horreur du sang "), mais si l'on examine attentivement la manière dont cet auteur analyse les sacrifices qu'effectue le maitre de la Terre pour réparer telle ou telle violation d'un tabou de la Terre, l'on s'aperçoit que ce qu'il cherche à articuler sur la finalité de ces pratiques rituelles ne va nullement dans le sens d'une théorie de l'expiation.

Le schème thérique autour duquel s'ordonne la recherche de Rattray sur la forme de déterioration qui affecte le pays après la violation d'un tabou de la Terre peut être énoncé ainsi : ce qui fait injure à la Terre dans la violation de tel ou tel de ses tabous, ce n'est pas en tant que telle la mise en contact de celle-ci avec une substance (sang, sperme, matière décomposée du cadavre), d'essence contraire à la sienne, c'est le fait que cette mise en contact se réalise en un lieu de l'agglomération villageoise qui, d'une manière ou d'une autre, fait toujours partie d'une aire rituelle et qui, à ce titre, est toujours susceptible d'entrer en connexion avec des lieux sacralisés, ceux qui, dans le cadre de cultes divers, reçoivent le sang des animaux sacrificiels. Il n'est pas possible de décrire ici les procédures qui sont mises en oeuvre en ces sociétés " voltaïques " pour articuler les unes aux autres les différentes unités territoriales dont se compose une localité soumise à la juridiction d'un même maitre de la Terre. Il nous suffira d'indiquer que si les habitants d'une même localité se distribuent en différents groupes de descendance - sections claniques ou lignages - qui ont, chacun, leurs propres cultes ancestraux, ils sont en même temps insérés dans les mailles d'un réseau de liens rituels qui font d'eux les 
" enfants d'une même terre ". Les sacrifices qu'effectue le maître de la Terre en diverses circonstances - soit auprès d'un autel de la Terre commun, soit en divers autres lieux de l'agglomération - mais aussi les sacrifices répétés que doivent assurer pour leurs propres unités territoriales les responsables de bosquets sacrés, font du territoire une sorte de tissu de liens sacrificiels. Rattray avait déjà commencé à mettre en place un concept que Meyer Fortes développera à propos d'une société de la même aire culturelle (les Tallensi), à savoir celui d'une parenté rituelle liant les membres d'une communauté qui sacrifient ensemble à une même " peau de la Terre". Il avait vu que les parents ainsi définis sont pris dans un champ dont tous les éléments - au premier abord hétérogènes - sont solidaires les uns des autres. Les tabous de la Terre et les sacrifices de réparation qui les accompagnent ne prennent sens qu'une fois replacés dans le cadre de cette sorte d'espace-corps qu'est une unité de maîtrise de la terre. Rattray a su comprendre que le maître de la Terre ne prononçait ses arrêts qu'en raison d'un savoir qu'il détient en propre, portant sur les justes partages des sangs sacrificiels répandus sur la terre. Il a pu ainsi entrevoir les criteres que ce personnage prend en compte pour discriminer ce qui dans les affaires de sang versé sur le sol faisait injure au culte rendu à la terre, provoquant ce que Mauss appelait une « coupure dans la toile du sacrifice" (Mauss, 1969a: 226). Nous ne pouvons nous étendre sur ce point, mais nous devons fortement souligner que dans les évaluations des « amendes » et des réparations sacrificielles qui font suite à une affaire de meurtre sanglant, le maître de la Terre doit prendre en considération toute une série de variables, comme par exemple, l'unité de maîtrise de la terre dont dépend le meurtrier et sa victime, ou encore l'identité sociale du responsable rituel de la portion de terre où le sang a coulé. En somme, on le voit, toute une casuistique. Tout se passe, en effet, comme si ce personnage devait d'abord déterminer quelles étaient les sphères d'appartenance sacrificielle des personnages impliqués dans une affaire de meurtre - et d'abord celles du meurtrier et de sa victime - avant de pouvoir décider si en cette affaire l'entame faite au corps d'un autre est aussi une entame faite à la terre. On est loin d'une logique de l'expiation. 
Si Rattray n'a pas exploré completement tous les problèmes que posent les riches documents qu'il a rassemblés sur la fonction sacrificielle qu'exerce en temps de meurtre le maître de la Terre, il a ouvert des pistes pour l'approche de cette question. Mais comme nous l'avons déjà indiqué, il a mené parallèlement des recherches sur la façon dont se présente l'activité sacrificielle lorsqu'elle se réalise dans le contexte de rites de réparation à caractère plus privé qui visent spécifiquement à traiter le mal dont est affligé le meurtrier et son entourage. C'est cet autre aspect de sa recherche que nous allons maintenant considérer en commentant les pages qu'il a consacrés à un rituel observé chez les Nankanse du nord du Ghana, le rituel dit tobega où la dimension sacrificielle passe au premier plan ${ }^{19}$.

\section{Un destin de meurtrier}

Le tobega - ou tubega - nous signale Rattray (1932: 310) au tout début de son propos " est cela même qu'une personne qui a tué " - soit qu'elle ait tué "à la guerre" (war), soit qu'elle ait commis un meurtre - « est dite acquérir en conséquence de son acte". Rattray ajoute que ce mot est aussi employé par métonymie pour désigner "l'autel " (shrine) qui est fabriqué pour un tel esprit (spirit) et aussi pour la cérémonie en relation avec cette fabrication (preparation).

$\mathrm{Si}$ énigmatiques qu'elles puissent paraître, ces formules de Rattray ${ }^{20}$ font bien apparaître que chez les Nankanse l'une des phases

19 Les Nankanse vivent à l'est de la Volta rouge de part et d'autre de la frontière qui sépare le Ghana (pays où ils sont principalement implantés) du Burkina Faso, où ils sont quelques milliers. Rattray indique que les membres de cette ethnie s'appellent

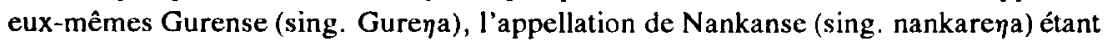
celle que leur donnent certains groupes kasena, leurs proches voisins. La langue parlée par ce peuple (famille mole-dagbane) est le gure peu arbitraire - mais c'est l'usage - nous dirons * un Nankana *, * des Nankanse *.

20 Stéphan Dugast a déjà fait une lecture du texte de Rattray consacré au tobega en vue d'une comparaison avec les rituels liés au meurrier, chez les Basar du Togo (Dugast : 1992). Cet essai d'analyse comparative nous a beaucoup servi pour notre propre travail de lecture. 
importantes du rituel qui est accompli pour traiter certaines catégories de meurtriers se présente comme une opération qui, par-delà l'acte matériel de fabrication d'un autel, vise à installer l'homme qui a tué dans le statut de desservant attitré d'un culte rendu à une puissance. Rattray est passé très vite sur certains aspects du rituel, mais il n'a pas surestimé l'importance des séquences qui sont centrées autour de l'autel du tobega. C'est bien le cycle de vie de cet autel qu'il faut suivre si l'on veut comprendre la dynamique propre du rituel en question.

Ce qui marque le début du procès, c'est la prise en charge du meurtrier par un personnage mi-prêtre, mi-magicien, spécialement qualifié pour mettre en place le type de rituel qu'implique une telle prise en charge. Les premiers gestes qu'effectuera ce spécialiste en présence du meurtrier sont des soins corporels à base de médecines et de bains, mais un examen attentif de cette première phase du rite montre que, dès ce stade, on traite le meurtrier non pas seulement comme la "victime " d'une sorte de mal contagieux dont il faudrait tout à la fois le protéger et protéger les autres, mais comme une sorte d'initiant appelé à devenir l'adepte d'un culte particulièrement contraignant. Dans les jours qui suivront ce premier traitement, on procédera a l'installation du tobega dans la maison du meurtrier. Après cette installation, les épreuves changeront de nature. L'état du meurtrier ne nécessitera plus, comme aux premiers jours, une prise en charge permanente, mais la vulnérabilité du sujet ne sera pas moindre qu'elle ne l'était durant la première période du traitement et cela en raison du fait que, dès cet instant, et durant toute sa vie, il devra demeurer dans la proximité de l'autel spécialement installé pour lui. Désormais, le meurtrier sera assujetti à la puissance tobega logée dans l'autel du même nom, puissance qui n'aura de cesse d'exiger de lui des sacrifices réguliers, sacrifices, nous le verrons, tout particulièrement astreignants, et sur la bonne exécution desquels d'anciens meurtriers, déjà traités, veillent. L'homme est pour ainsi dire sous surveillance. Il le restera toute sa vie. Après sa mort, la puissance qu'il aura entretenue restera active et cela jusqu'au moment où l'on célébrera pour lui la cérémonie finale des funérailles. Alors d'anciens meurtriers, détenteurs d'autels du même type, viendront enlever l'autel pour le mettre en un lieu où 
les effets destructeurs de la puissance ne pourront plus s'exercer sur la maison de ses héritiers.

Ce résumé succinct des faits nous montre que chez les Nankanse l'acte du meurtre déclenche toute une série de séquences d'actes qui s'échelonnent sur une longue période de temps, période dont la durée déborde le temps de vie du meurtrier. Certes, il y a quelque artifice à vouloir repérer dans la suite des opérations effectivement réalisées des phases bien distinctes car le chemin du rite n'est pas rectiligne. $\mathrm{Si}$ sinueux que soit ce chemin, on peut tenter de le reparcourir en prêtant attention aux métamorphoses successives de la puissance qui a été libérée par le meurtre. Dès lors qu'on adopte une telle perspective, on ne peut pas ne pas remarquer que ce qui rythme le temps du rite, c'est la nécessité de circonscrire dans les limites de l'espace cultuel de l'autel du tobega le champ d'intervention de la puissance.

Mais avant de développer ce point, il nous faut dire quelques mots sur la façon dont ont été recueillis les faits dont nous allons faire état.

C'est au chapitre XII de The Tribes of Ashanti Hinterland qu'il est pour la première fois fait mention des croyances et des rites liés au tobega ; toutefois, le véritable auteur de ce chapitre n'est pas Rattray mais V. Aboya, le collaborateur nankana de Rattray ${ }^{21}$. En ce chapitre, Aboya poursuit sa chronique de la vie rituelle quotidienne des Nankanse par un récit des coutumes suivies lors des funérailles d'un Ancien. En un passage de ce récit, Aboya nous décrit les conduites que les héritiers du mort doivent exécuter pour traiter les "possessions "

21 Durant son enfance, V. Aboya avait été " vendu comme esclave *. Après diverses aventures, il retrouva sa liberté, fut recueilli par des missionnaires protestants et c'est dans ce milieu d'accueil qu'il apprit à lire et à écrire. Après quelques années, il retourna dans son village natal et c'est là qu'il fit un jour la connaissance de Rattray. L'ethnologue lui demanda de consigner par écrit, et dans les termes de sa propre langue, des scènes de la vie quotidienne s'étant réellement produites dans son village. Ce sont ces récits d'Aboya (des * pen pictures of native life in a nankanse village *), traduits par Rattray (les originaux sont en gurene), qui constituent l'essentiel des chapitres 5 à 16 de la monographie, ceux qui ouvrent la section consacrée aux Nankanse. Ce mode de présentation reflète un choix de méthode : montrer au lecteur les coutumes d'un peuple en lui faisant d'abord connaître le point de vue de ceux qui vivent ces coutumes de l'intérieur et qui les pensent à travers les mots de leur langue maternelle. 
et les " autels " laissés par le défunt, et c'est dans ce contexte que surgit la question des coutumes spéciales qu'on doit accomplir quand l'autel à traiter n'est pas un autel ordinaire, mais un tobega. Interrompant pour un temps le fill de son récit, Aboya juge nécessaire de donner quelques informations sur la façon dont cet autel a été constitué du vivant du meurtrier. Au chapitre XXVIII, la question du tobega est a nouveau abordée, mais cette fois sous la forme d'une petite note de synthèse faite par Rattray lui-même.

Les deux textes - celui d'Aboya et de Rattray - ne font pas double emploi. Bien qu'on y trouve quelques indications sur le rituel qui ne figurent pas dans le texte d'Aboya, le texte de Rattray se présente surtout comme une sorte d'explicitation des propos - souvent fort laconiques - de son principal collaborateur ou de quelques autres informateurs de la même ethnie. Dans les pages qui suivent, nous nous appuierons sur les deux textes mais pour la chronologie du rituel, c'est le texte d'Aboya qui nous servira de texte de référence. Ce que nous livre Aboya, ce n'est pas un rituel type, mais certaines séquences d'un rituel réellement observé - par lui-même ou par certains de ses proches - dans le contexte d'une affaire de sang où le meurtrier était un familier (sans doute un homme du même village). Tout à son récit, Aboya ne cherche pas à donner une définition abstraite des différentes catégories d'homicides qui devaient passer par l'épreuve du rituel du tobega, mais il ressort de l'une de ses formules que dans l'optique de son groupe, on englobait dans un même ensemble - celui des gens soumis à l'obligation du traitement - ceux qui avaient tué à la guerre, mais aussi certains meurtriers. Pour quelles sortes de « tueurs " furent organisées les performances rituelles qu'il s'attache ensuite à décrire ? Un guerrier ou un meurtrier ? On peut inférer du compte rendu qu'il nous donne d'un épisode du rituel que la performance observée s'est réalisée dans le contexte d'une situation où l'homme qui avait tué l'avait fait délibérément lors d'une expédition de représailles lancée contre des gens d'une autre localité. A une étape du rituel, nous dit-il, un cortège fait d'anciens « tueurs " s'éloigne du village où a été effectué le traitement et « descend dans la vallée » jusqu'à l'endroit précis où l'homme qu'on est en train de traiter, quelque temps auparavant, avait décoché sa flèche mortelle. Sous la plume d'Aboya, l'expression 
" descendre dans la vallée " renvoie tantôt à une situation d'expédition guerrière, tantôt à une action de vengeance lancée contre une personne - ou un groupe - qui ne sont pas inclus dans le réseau des liens claniques auquel appartient le vengeur. Elle ne s'applique donc pas au mouvement d'impulsion qui, en certaines circonstances, pousse un homme à frapper à mort un parent ou une personne qui vit sur la même terre. Bien qu'il ne s'explique pas vraiment sur les suites qui étaient données à une affaire de sang de ce type, Aboya prend soin de préciser qu'on procédait également en ce cas à la célébration d'un tobega. Sur ce point Rattray est encore plus net qu'Aboya, car lorsqu'il parle des personnes qui étaient soumises à un tel rite, il ne fait pas de différence entre celles qui " avaient tué à la guerre " et celles qui avaient commis une sorte d'assassinat. En outre, il fait état d'un rituel tobega célébré pour un meurtrier qui avait tué une femme. Est-ce à dire qu'à la suite de pareils meurtres, le rituel qui était effectué se déroulait de la même façon que celui qui était mis en place pour un homme «descendu dans la vallée »? On a quelques raisons de supposer qu'il devait s'en différencier de quelque manière. Ceci une fois précisé, nous pouvons maintenant entrer dans l'analyse d'un rituel dont l'événement déclencheur est le retour en son village d'un homme qui, dans le contexte d'une action de représailles, vient de répandre le sang d'une personne habitant une autre localité que la sienne.

Nous allons conduire cette analyse en nous arrêtant sur quelques moments du procès qui nous paraissent particulièrement significatifs. Pour l'un de ces moments, celui qui concerne "la pose du dogele ", l'information donnée par Aboya est si ténue que nous n'aurions pas grand chose à en dire si nous devions être entièrement tributaire du récit d'Aboya. Grâce aux textes que D. Liberski et $S$. Dugast ont écrit pour le présent recueil, nous avons pu rétablir certains chaînons manquants du mécanisme mis en jeu lors du déroulement de cette phase. A propos des Kasena du Burkina Faso et des Bassar du Togo, ces deux auteurs ont décrit par le menu des séquences rituelles liées au traitement d'un meurtrier qui, par bien des traits, sont extrêmement proches de la séquence nankana dite « pose du dogele». Le moment venu, nous 
citerons ces deux auteurs pour justifier ce que nous avancerons sur la signification de cette séquence 22 .

\section{La perception de l'événement, les premiers symptômes du mal et la recherche du spécialiste}

«Quand ils voient que le sang a frappé un homme, ils courent chez celui qui est en possession des racines". C'est en ces termes qu'Aboya tente de restituer la perception de l'événement qui va déclencher les premiers gestes du rituel. On remarquera que ce n'est pas «l'homme qui a le sang» (c'est ainsi que le narrateur désigne le meurtrier) qui prend de lui-même l'initiative de ces premiers gestes. Le sujet pluriel de la phrase d'Aboya renvoie sans doute à des gens de sa maison ou faisant partie de son entourage proche. Ce sont ces gens qui dans l'instant même où ils rencontrent l'événement (le sang qui a frappé un homme), se précipitent chez l'homme qui détient « les racines ", celui-là même qui va conduire le rituel. Le fait que la démarche à entreprendre auprès de cet homme soit placée sous le signe de l'urgence (ils courent) n'est pas un trait spécifique du rituel nankana. Les témoignages qu'on a pu recueillir en d'autres populations voltaïques sur des rituels comparables mettent aussi l'accent sur cette exigence d'agir vite, et cela comme si à la rapidité du geste qu'avait dû porter le meurtrier pour frapper à mort sa victime faisait écho la rapidité des coups que la puissance mortifere éveillée chez sa victime allait porter contre lui et ses proches. Aboya passe assez rapidement sur la façon dont les Nankanse décrivent les premiers symptômes du mal qui sont susceptibles d'atteindre le meurtrier en son corps et en son psychisme, mais les formules qu'il emploie pour restituer ce qui se dit en cette ethnie sur les malheurs virtuels du meurtrier ou sur la

22 Certaines des pratiques rituelles qu'observent ces trois sociétés de l'ensemble voltaique offrent tant de traits de ressemblance qu'elles apparaissent presque indiscernables. Les Nankanse sont des voisins directs des Kasena. Selon D. Liberski, les Kasena sont très conscients de ce qui les rapproche de leurs voisins dans leurs façons de traiter les affaires de meurtre. 
nature des risques qu'encourent ses proches sont assez éloquentes : " si vous ne faites pas cela, cela vous tuera ou tuera les gens de votre maison ou vous mourrez brusquement sans avoir été malade ». Rattray ne dit pas autre chose, mais précise que, sans traitement, c'est « la personne tuée " qui " vous conduira à la folie ". A propos du style de conduite que doivent adopter certains meurtriers dans les jours qui ont suivi leur acte, Rattray nous apporte des informations qui nous dévoilent un aspect essentiel du désordre mental dont le meurtrier, entre autres maux, est censé être atteint. Il nous dit que lorsque la victime du meurtre est une femme, celui qui l'a tué « doit passer l'épreuve qui consiste à simuler tous les travaux habituellement faits par une femme " (Rattray, 1932 : 311).

Quel est donc ce personnage chez qui on accourt sitôt après le meurtre pour s'occuper du meurtrier ? Les renseignements que nous donnent Aboya et Rattray sur le personnage en question sont très fragmentaires (en particulier, ils ne nous disent pas si ce personnage devait ou non appartenir au lignage du meurtrier), mais si l'on replace ces renseignements dans l'ensemble des données qu'ils nous ont fournies sur la façon dont les Nankana sélectionnaient leurs prêtres, leurs magiciens et leurs guérisseurs, on peut se faire une idée assez précise de son statut rituel. Ce qui ressort nettement des textes, c'est le fait suivant: l'homme qu'on allait trouver en cette circonstance devait nécessairement être sélectionné dans un ensemble de personnes qui, à un moment de leur vie, avaient été affectées par la même sorte d'affection que celle dont était présentement saisi le meurtrier. Rattray le répète à plusieurs reprises : ne pouvaient accéder à la position de spécialiste habilité à traiter ce genre d'affection que d'anciens meurtriers d'abord passés par l'épreuve du rituel du tobega et qui, après cette épreuve, avaient changé de statut en s'engageant dans la voie d'une autre forme d'initiation. Rattray ne décrit pas cette initiation, mais on peut penser qu'elle prenait la forme d'une série d'épreuves liées à l'acquisition d'une catégorie particulière « d'autel ". C'est bien en tout cas la possession de cet autel qui faisait accéder l'ancien meurtrier à une position de maîtrise dans la conduite du traitement rituel des nouveaux meurtriers. 
L'expression qui vient parfois sous la plume de Rattray pour qualifier ce genre de spécialiste est celle de witch doctor. L'expression peut surprendre, mais l'on peut aisément comprendre les raisons qui ont conduit Rattray à l'employer. Il existait en effet chez les Nankanse toute une série de witch doctors à qui l'on confiait la direction de rituels complexes et qui se trouvaient habilités à le faire en raison d'un principe de qualification qui parfois n'etait pas très different de celui qui présidait à la sélection du spécialiste de l'affection liée au meurtre. Ce qui, en effet, qualifiait ces différents spécialistes, c'est le fait qu'ils étaient censés avoir fait l'expérience du même type d'affliction que celui dont était victime leur patient. A cette catégorie de spécialistes était associée l'image d'une forme de pouvoir évoquant tout à la fois celui du magicien et celui du prêtre. Pouvoir de magicien en tant qu'on leur attribuait la possession d'une sorte de patrimoine reçu en legs et " jalousement gardé ", comprenant tout a la fois des "médecines " d'origine végétale (en particulier des racines), un stock de connaissances secrètes (relatives aux plantes, aux recettes, aux formules à réciter), mais aussi tout un ensembie de choses impalpables passées de maître à élève au fil des années d'apprentissage. Pouvoir de prêtre en tant qu'on leur assignait la position d'agent principal d'un culte sacrificiel rendu à une puissance auprès d'un autel déterminé. Au chapitre XXVII de son ouvrage, Rattray dresse une liste impressionnante de rituels magico-religieux dont l'exécution était confiée à ces sortes de prêtres-magiciens, rituels à finalités diverses mais qui avaient pour commun dénominateur de contraindre le cours des choses à répondre aux objurgations faites à la puissance présente en leur autel, et cela dans des situations de crise bien circonscrites échappant à la compétence du commun des mortels livrés aux seules ressources des médecines propres de leurs lignages. Dans cette liste, on ne trouve pas seulement des rites thérapeutiques, mais aussi des rites visant à lever des enchantements pour des impuissances de toute nature provoquées par les hommes ou les dieux.

Quand on lit la description de certains de ces rituels, on constate que les procédures mises en cuvre se déploient toujours autour d'un foyer central, appelé "medicine shrine " par Rattray, sorte d'autel sacrificiel régulièrement entretenu, servant d'habitacle à une puissance 
dûment nommée et se présentant le plus souvent sous l'aspect de poteries emplies d'une substance liquide où trempent souvent des racines, ainsi qu'un certain nombre d'objets et d'ingrédients de toutes sortes. Il semble bien aussi que ce qui fait l'unité de tous de ces rites, c'est la présence d'un schème d'action assez uniforme. Sitôt connu l'événement déclencheur, des gens directement concernés par l'issue du traitement (pas nécessairement des parents du sujet affecté) iront trouver en son domicile le spécialiste de l'affection. L'homme ainsi sollicité n'acceptera d'intervenir qu'après avoir reçu des offrandes sacrificielles (de la bière et des victimes animales), celles qui conviennent à la puissance qu'il dessert. Il y aura ensuite la quête de « racines " d'espèces végétales déterminées. Celles-ci une fois réunies, on pourra alors procéder à l'exécution de la première phase du traitement souvent désignée par l'expression "la pose de la poterie ", traitement qui impliquera souvent l'enchaînement des mêmes actes, a savoir : une installation en un lieu du traitement d'une ou de plusieurs poteries ; une mise en place sur un foyer spécial de la poterie emplie d'un mélange liquide où baignent des fragments de racines et des ingrédients de toutes sortes; des sacrifices et des libations sur la (ou les) poterie(s) avec invocation de la puissance tenant en son pouvoir le sort du patient; une opération de cuisson jusqu'à ébullition de la décoction ; des bains ou des aspersions répétés du sujet avec le liquide utilisé pour la cuisson. Bien que non nécessairement présentes dans tous les rites de ce type, seront également réalisées assez souvent les séquences suivantes : une série d'opérations faites sur d'autres racines avec carbonisation et réduction en poudre ; absorption par le sujet traité, ou par certaines personnes qui l'assistent, d'une nourriture ou d'une boisson mélangées avec la poudre ainsi obtenue. Ce qui marquera parfois la fin de cette premiere phase du traitement, ce sera un acte visant à opérer une mise à distance du sujet traité d'avec ce qui constitue le « reste " des " racines " utilisées pour le traitement. 


\section{La pose du dogele pour le meurtrier}

Si bref que soit le récit que nous donnent Aboya et Rattray de la premiere phase du traitement auquel on soumet le meurtrier - il se réduit à quelques phrases - il contient assez d'indications pour qu'on puisse y retrouver les principaux éléments du scheme que nous venons de présenter. Les citations suivantes en témoignent.

Lors de la première démarche faite auprès de l'homme qui possède les racines, il faudra d'abord lui donner comme présents, ainsi parle Aboya, « des poulets, un chien et de la bière ». Lorsqu'arrivera le moment du traitement, précisent ensuite nos deux narrateurs, ils devront " couper les racines " (Rattray) et les « cuire... jusqu'à ce que l'eau bout " (Aboya). Ensuite, ils prendront une calebasse, la rempliront de l'eau médicinale bouillie contenue dans la poterie et « la verseront sur la tête de celui qui a le sang ", et cela trois fois de suite. "Ils feront ainsi pendant trois jours "(Aboya). Avant d'être soumis a cette epreuve, le meurtrier aura les cheveux rasés (Rattray). Le dernier acte de cette première phase de traitement, Aboya en rendra compte dans les termes suivants : " Le jour où ils jettent les racines, < ceux qui connaissent l'eau $>$ (sic), se réunissent en nombre, et emportent les racines en chantant en chour (sakera) pendant qu'ils descendent vers la vallée, de la même façon qu'ils l'ont déjà fait quand les hommes sont partis s'entre-tuer. Là, ils jettent les racines ". 23

A quelques détails près, ce schème est très proche de celui que décrivent ici $S$. Dugast et $D$. Liberski dans la partie de leurs textes où ils rendent compte des premiers traitements que devront recevoir les meurtriers bassar et kasena. D'une ethnie à l'autre, on est frappé par l'étroitesse des similitudes : un même type de dispositif matériel (un foyer spécial, une poterie ou un chaudron empli d'une mixture complexe), une opération de cuisson, une utilisation de plusieurs sortes de

23 Il est probable que l'expression * ceux qui connaissent l'eau * renvoit au même type d'institution initiatique que décrit ici D. Liberski (1991) à propos des Kasena. Si tel est le cas, * ceux qui connaissent l'eau * seraient donc non pas nécessairement des meurtriers, mais des hommes originaires du même village que le meurtrier, et qui auraient connus ce type d'initiation. 
racines, des traitements externes du corps du patient (aspersions et bains). Un trait qui n'apparaît pas clairement chez les Nankanse, mais qui en revanche est fortement marqué chez les Kasena et chez les Bassar, c'est l'aspect sacrificiel de toute l'opération. Pendant le déroulement du rite, des paroles sont prononcées qui se présentent comme des invocations faites à une puissance, comme si l'efficace des gestes allait devoir dépendre du vouloir de la puissance ainsi interpellée au moins autant que du degré de savoir-faire du witch doctor dans l'application de ses connaissances médicinales et de ses recettes magiques. Mais il y a plus. Dans les deux cas, les invocations faites à la puissance prennent place au sein d'une suite d'operations où, comme le fait remarquer Dugast, s'imbriquent étroitement procès sacrificiel et préparation de médecines. La dimension sacrificielle de la relation qui est nouée ou renouée avec cette puissance est elle-même (et dans les deux ethnies) un trait marquant du rite, le spécialiste y jouant le rôle de prêtre-sacrificateur, le meurtrier, celui de sacrifiant. Chez les Kasena, c'est un rite sacrificiel qui inaugure l'opération de cuisson car le feu n'est mis sous la poterie qu'après que le cad-tu y ait sacrifié un chien et un poulet fournis par le meurtrier. Chez les Bassar, c'est au-dessus de l'endroit où a été immolé l'animal et où sont entassés les objets de la puissance que le meurtrier et le jabun devaient faire tournoyer la marmite avant de pouvoir la déposer sur le foyer. Bien qu'Aboya, dans son compte rendu de la premiere phase du rituel, ne fasse pas explicitement état d'un sacrifice de ce type, l'on a de bonnes raisons de supposer que dans ce cas aussi, c'est par un sacrifice que s'effectuait la mise en place sur le foyer de la poterie dogele. Ce qui nous autorise à faire cette hypothèse, c'est le fait que dans la plupart des rites de la même famille, le maître du rite, avant même qu'il ne se livre à l'opération de cuisson, devait d'abord verser le sang du sacrifice sur la poterie-autel, et cela à partir d'une victime animale fournie par le patient ou par ses proches. Va également dans le sens de cette hypothèse une brève indication donnée par Aboya au début de son compte rendu. Il nous signale, en effet, que les proches du meurtrier, lors de leur première démarche auprès du spécialiste, ne se présentent pas à ce dernier les mains vides, mais avec, comme "présents ", des poulets, un chien et de la bière. Ces choses données au spécialiste, on ne 
voit pas quelle autre finalité elles pourraient avoir que celle d'entrer dans le rituel au titre de victimes et d'offrandes sacrificielles données a la puissance au nom du meurtrier. Cette supposition nous paraît d'autant plus plausible que dans la liste des choses données figure un animal (le chien) dont nous savons par de nombreux témoignages D. Liberski le rappelle -- que dans les rituels "voltaïques " liés au meurtre, il prend souvent valeur d'emblème pour marquer la position singulière d'un meurtrier.

Interprétées à la lumière des donnés recueillies chez les Bassar et les Kasena, les formules extrêmement laconiques d'Aboya prennent donc un sens qui n'apparaissaient pas en premiere lecture. Elles laissent entrevoir que le traitement auquel les Nankanse soumettent le meurtrier dans les jours qui suivent l'effectuation de son acte est une opération qui met en jeu une relation à plusieurs termes où se trouvent pris, non pas simplement un soignant et un soigné, mais un soignant qui est aussi un prêtre sacrificateur, un soigné occupant la position d'un sacrifiant, et une puissance. L'espece d' " alchimie " à laquelle se livre le spécialiste du rite n'est pas séparable de l'opération sacrificielle par le moyen de laquelle ce même spécialiste éveille et met en activité la puissance. C'est de cette puissance qu'on attend une transformation de l'état dans lequel se trouve le meurtrier.

De la puissance - dite jabun - que le spécialiste bassar, au cours de « la cure », éveille et contrôle, S. Dugast, s'appuyant sur les dires de ses informateurs, nous dit d'emblee qu'elle aurait pour vocation première de lutter contre le kinan - le principe vital - de la victime, principe vital qui, dès l'instant du meurtre, chercherait à s'introduire dans le corps du meurtrier. S'appuyant sur les paroles que prononce le càà-tu au moment où il sacrifie le chien qui a été fourni par le meurtrier, D. Liberski, s'interrogeant sur la nature de la puissance qui intervient lors du rite kasena, se sert d'une formule qui la fait apparaître comme l'instance qui est susceptible de repousser les assauts répétés de la personne tuée, assauts dont le meurtrier est la proie. A partir de ces premiers constats, ces deux auteurs analysent de façon approfondie le lien qui unit l'une à l'autre, dans le rite, la puissance qui, émanant de la personne tuée, maintient sous son emprise le meurtrier, d'une part, cette autre puissance par le moyen de laquelle va 
s'opérer une mise à distance du meurtrier et de sa victime, d'autre part. Lorsque nous analyserons la relation qui s'engage entre le meurtrier et son tobega, nous serons amené à soulever un même type de questions, mais à cette étape de notre description, il serait hasardeux de le faire car nous ne savons presque rien de la nature de la puissance qu'active le spécialiste à cette phase du rituel dite « pose du dogele ". Le compte rendu d'Aboya n'indique rien d'essentiel sur la relation à la puissance ; ce qu'il livre, en revanche, sur la façon dont les Nankanse se représentent l'opération de cuisson faite sur les racines est particulièrement éclairant.

Lorsqu'Aboya évoque pour la première fois l'épreuve rituelle que devra subir l'homicide, il se sert de l'expression suivante : "Après le combat vous avez bouilli, la personne que vous avez tuée». C'est a cette phrase que fait directement suite la remarque que nous avons déjà citée : « si vous ne faites pas cela, cela vous tuera ou tuera les gens de votre maison ou vous mourrez brusquement sans avoir été malade". Une telle phrase jette un éclairage inattendu sur la portée du geste d'aspersion plusieurs fois répété (trois fois de suite pendant trois jours) que le spécialiste effectue en direction du corps du meurtrier - geste, nous l'avons vu, qui consiste à verser sur la tête de "celui qui a le sang ", une eau de racines sortie bouillante de la poterie où elle a été mise à «cuire ». Le meurtrier est bien de fait « ébouillanté", mais l'expression relevée par Aboya nous invite à penser qu'à travers cette opération drastique, ce qu'on cherche à produire comme effet, ce n'est pas seulement une transformation de l'état du meurtrier, mais un changement d'état de sa victime. Que la victime du meurtre soit bien là d'une certaine façon présente dans le traitement auquel on soumet son meurtrier et qu'elle y soit non pas à la manière d'un esprit qui rôde, mais au titre d'occupant d'un lieu qui est le lieu même qu'occupe le corps du meurtrier, c'est une hypothèse qui nous paraît d'autant plus soutenable qu'elle s'accorde bien avec l'épisode final de la première phase du traitement, lequel, rappelons le, consiste à aller jeter les racines utilisées pendant le traitement au lieu même où l'homme qui a été mortellement fléché est tombé. Chez les Bassar et les Kasena, les racines qui ont servi au traitement du meurtrier seront, après usage, mises en un lieu où ce qu'elles recèlent encore de puissance contenue 
sera pour ainsi dire neutralisé (on les jette à un carrefour, ou bien on les enterre). Le fait que chez les Nankanse l'on revienne, aussitôt le traitement fini, sur le lieu du meurtre pour jeter les racines suggère que ce qu'on jette avec celles-ci, c'est quelque chose qui, en venant prendre la place qu'occupa un temps le corps gisant de la victime, viendra porter témoignage de ce qu'est devenu le corps de celle-ci, une fois achevé le premier traitement du meurtrier.

On est ainsi conduit à l'idée que l'économie du traitement auquel est soumis le meurtrier repose toute entière sur la croyance que ce qui de la personne, victime d'un meurtre, s'est logé dans le corps de son meurtrier, c'est une chose encore bien vivante, une chose à certains égards comparable à ce qu'on se représente de la puissance de ces morts qui n'ont pas encore fïni de mourir, tels qu'on les met en scène à certaines étapes des rites funéraires, par exemple en ces moments de confession publique où c'est le cadavre du mort, par le mouvement même que lui imprime ses porteurs, qui vient désigner celui ou celle qu'il accuse. Certes, on ne peut pas comparer terme à terme ce qu'on prête à la vindicte des morts dans le temps des rites funéraires avec ce qui se dit autour de ce mort singulier qu'est la victime d'un meurtre, mais il semble bien en tout cas que le traitement rituel du meurtrier nous mette en présence d'une même forme de passage que celle qu'on peut observer dans les rites funéraires. Dans les deux cas, ce qu'il faut assurer pour le mort, c'est le passage d'un état où il a gardé les attributs d'un vivant à un état de mort réalisé, on pourrait dire à un état de mort enfinn cadavérisé. Cette mise en regard du traitement du meurtrier avec le rituel funéraire peut aussi éclairer le geste final du dépôt des racines bouillies dans la vallée du meurtre. Alors qu'ils auront déjà - et depuis longtemps - donné à leur mort sa sépulture, les parents du disparu, lors des nombreuses séquences qui dans un cycle funéraire ordinaire suivent l'enterrement, devront plusieurs fois manipuler un simulacre du cadavre, comme si le corps mis en terre n'était pas encore suffisamment cadavérisé et qu'il fallait encore beaucoup de temps et beaucoup de manipulations de ce type pour qu'on soit assuré que le disparu ne reviendrait plus parmi les siens comme un mort " vif ", un mort aussi vif qu'était vif son cadavre lors des séances de confession publique. Les racines bouillies jetées dans la vallée du 
meurtre pourraient bien être comparées à l'un de ces simulacres de cadavre si souvent présents dans les rites funéraires. Manipulé dans ce nouveau contexte non par les gens du deuil, mais par des personnes qui font cette fois partie du cercle des proches du meurtrier, ce simulacre viendrait porter témoignage du processus de transformation qui a été opéré sur la victime, la faisant passer d'un état où, s'étant insinuée dans le corps du meurtrier, elle y conservait sa vie à un état où, séparée de ce corps, elle serait devenue la victime d'une mort enfin réal isée.

On retrouve trace d'une pareille conception en d'autres populations voltaïques. A propos du traitement du meurtrier tel qu'il l'a étudié chez les Lo-Dagaa (une population "voltaïque " du nord du Ghana), Goody note que la seule eau que doit boire le meurtrier à la phase active du traitement est une eau dans laquelle « the bones of a victim have been boiled». Ici, le spécialiste détient en réserve une pièce anatomique prélevée sur le cadavre d'un homme également victime d'un meurtre et c'est cette pièce qu'il fait bouillir pour préparer le breuvage du nouveau meurtrier qu'il a en traitement. Bien qu'on ne retrouve pas chez les Lo-Dagaa une métaphore semblable à celle que fournit Aboya, le fait cité nous met en présence du même type d'alchimie. L'opération n'a pas pour but, selon nous, de faire ingérer par le meurtrier une propriété de la victime - sorte de valeur ajoutée - qui serait contenue dans une partie de son squelette, mais de permettre que se poursuive par le moyen de cette ingurgitation un processus d'épuisement de ce qui reste de vie de la victime dans le corps du meurtrier.

Mais cette notion d'épuisement à laquelle nous confronte le traitement du meurtrier vu à la lumière du traitement du mort dans les rites funéraires ne permet pas de rendre compte de ce qui constitue en propre l'état du meurtrier avant le traitement. Certaines formes de conduites de deuil, et en particulier des conduites de mortification, semblent indiquer - Hertz l'avait bien montré - que ce qui se réalise pour certains parents du mort, c'est une forme d'identification au mort. Qu'il puisse y avoir en pareil cas une relation avec le mort qui soit aussi proche que celle qui lie le meurtrier à sa victime, rien ne nous permet de le dire et cela ne ressort guère des données observées. Dans 
un texte de G.F. Hegel, texte qui porte spécifiquement sur la vengeance, l'on trouve une formule dont la portée heuristique n'est peut être pas aussi grande que celle qu'imaginait le philosophe, mais qui met bien l'accent - et en des termes saisissants - sur la façon dont le meurtrier est pris par sa victime. Après avoir développé l'idée d'un meurtre qui opérerait à la manière d'une négation, Hegel ajoute aussitôt que cette "vie qui a été niée " par le meurtre, loin d'avoir été " supprimée par cette négation », continue à "subsister ", et cela, précise-t-il, non comme un « esprit immatériel », mais comme « une vie restaurée " qui effectue " l'inversion de ce qu'avait posé le meurtrier » (produisant «contre lui une attaque ") ; et le philosophe de conclure : « ce qui donc continue à subsister, ce n'est pas un esprit mais un esprit auquel le meurtrier a procuré un corps " (Hegel, 1976 : 155-156). Ce qui est intéressant pour nous dans cette formule, c'est la notion de vie restaurée. Quant à l'hypothèse que le meurtrier viendrait procurer un corps à l'esprit de la victime, elle a pour nous l'inconvénient d'être trop générale. Si certains traits de conduite prêtés au meurtrier avant sa cure évoquent bien une forme de possession, le concept de possession est trop large pour pouvoir être appliqué au cas qui nous occupe. D. Liberski, à propos des Kasena, fait l'hypothèse qu'en cette ethnie, ce qui est traité dans le rituel, ce n'est pas seulement l'acte d'avoir ôté une vie, mais tout autant le fait que l'agression meurtrière a pris la forme d'un fléchage mortel avec effusion de sang provoquant ainsi une forme de mort qui dans la symbolique de l'ethnie confere à la victime un statut de mauvais mort. Cette hypothèse éclaire bien des données ethnographiques recueillies dans toute une série de sociétés voltaïques, mais comme les faits nankanse sur cet aspect du problème du meurtre ne sont pas des plus parlants, nous ne la mettrons pas ici en discussion.

\section{Le rite d'installation du tobega}

Entre le moment où l'on dépose dans * la vallée " les racines bouillies et celui où l'on installe pour le meurtrier l'autel dit tobega, on ne marque pas, semble-t-il, de temps de pause. Les deux actes 
semblent devoir s'enchaîner. Dès leur retour au village, les proches du meurtrier iront retrouver le spécialiste - l'homme qui a déjà posé le dogele - pour lui demander de prendre en charge la suite du rituel. Aux hommes venus le trouver, le spécialiste confiera l'exécution d'un certain nombre de tâches dont certaines sont de même nature que celles dont il les avaient chargées à la veille de la pose du dogele : une fois de plus, il leur faudra faire préparer de la bière et se mettre à la recherche d'animaux sacrificiels présentant des caractéristiques bien déterminées (en l'occurrence la recherche d'un chien rouge, d'un coq rouge et d'une poule noire). Mais une démarche supplémentaire leur sera aussi confiée qui caractérise en propre cette phase préparatoire : ils devront aussi se procurer la corne d'un bélier noir, d'une part, la peau d'une variété d'écureuil, d'autre part, ces deux pièces devant fournir les deux composantes essentielles du futur autel. Ces pieces animales sont-t-elles déjà disponibles, gardées dans quelque réserve, ou faudra-t-il que les proches du meurtrier aillent « abattre » des animaux de l'espèce désignée pour prélever sur leurs corps les pièces en question? Ce point reste indéterminé comme l'est également la variété d'écureuil qui est exigée pour ce type de rite. Rattray nous signale que dans la langue courante, tobega est bien le terme dont on se sert pour désigner une variété d'écureuil ${ }^{24}$. Sur la base de cette indication, on est en droit de supposer que l'autel du meurtrier est porteur d'attributs qui renvoient à l'espèce en question. Tout se passe comme si un tel autel était placé sous le chiffre de l'écureuil, et cela, d'une double façon: de par son nom, d'une part, de par la provenance des matériaux dont il est fait, d'autre part. Rattray nous apprend qu'il existe chez les Nankanse une autre variété « d'autel de meurtre » qui de par sa structure matérielle et de par son nom renvoie aussi à l'écureuil. C'est l'autel dit tuko réservé à des gens qui n'ont pas eux-mêmes effectué un meurtre, mais qui sont impliqués dans une affaire d'homicide. Tobega et tuko renvoient à deux espèces différentes d'écureuil et l'on peut donc imaginer que, d'une espèce à l'autre, il y a des

24 Des populations voisines des Nankanse désignent par le terme de tobog l'autel lié au meurtrier. Il s'agit surement d'un emprunt mais Rattray ne nous dit pas si tobog, dans la langue des emprunteurs, est aussi le nom qu'on donne à l'écureuil. 
différences qui sont " bonnes à penser " pour figurer des différences de statut entre plusieurs catégories d'homicides.

Au jour fixé pour l'installation de l'autel, le spécialiste se rendra dans la maison du meurtrier où se sont déjà regroupés les personnes qui devront prendre part au rituel. Tout ce qui a été demandé par le spécialiste - la peau sac de l'écureuil, la corne d'un bélier, la bière et les animaux sacrificiels - a été amené sur les lieux et le spécialiste peut maintenant entreprendre la série d'opérations qu'impliquent tout a la fois la mise en place d'un nouveau tobega et l'installation en son nouveau statut de celui qui en sera désormais l'officiant attitré. Le spécialiste a amené avec lui de nouvelles racines. Préparer ces racines en vue d'en tirer une nouvelle substance, telle sera sans doute l'opération qui marquera le début du rite. En cette phase, ce qu'il s'agit d'obtenir, ce n'est plus une décoction mais une poudre noire, celle qu'on tirera des racines après carbonisation. Selon Aboya (Rattray ne dit rien sur cet aspect du rite), un peu de la poudre obtenue après carbonisation des racines sera introduit en ces deux réceptacles que sont le sac fait en peau d'écureuil, d'une part, la corne de bélier, d'autre part. Dans son descriptif, Rattray mettra surtout l'accent sur la séquence sacrificielle, et cela comme si il voyait en chacune des opérations de cette séquence ce qui va sceller le lien à la puissance. Selon cet auteur, la première de ces operations est une libation faite sur la peau et sur la corne par versement d'un peu de bière et d'un peu d'eau de farine. Il faudra ensuite sacrifier le coq rouge et la poule noire. Il faudra enfin procéder à la mise à mort du chien rouge. Lors de l'immolation de cette troisième victime, le spécialiste exhortera à haute voix la puissance tobega en prononçant les mots suivants : « un tel ou un tel t'a tué, si tu es une mauvaise personne, pars loin et laisse le seul ". Cette dernière opération une fois effectuée ${ }^{25}$, on devra assembler des pieces de l'autel. Selon Rattray, il suffira pour cet assemblage de lier ensemble, outre la peau et la corne, un troisième élément pris sur le corps du chien offert en sacrifice (en l'occurrence la queue de

25 Il est probable que ce premier sacrifice devra être suivi d'un acte de consommation des viandes sacrifielles, acte qui nous le verrons est un aspect essentiel du lien avec la puissance. 
cet animal). Ce geste fait, le spécialiste fera rentrer l'objet ainsi obtenu dans cet abri à usage rituel que les Nankanse appellent le zono, un petit édifice attenant au parc à bétail qu'on trouve dans chaque maison d'habitation. Détail significatif : l'objet ne sera pas posé sur le sol, mais suspendu à l'une des poutres du toit du zono. Ce dernier geste semble marquer la clôture de cette phase du rituel.

Le tobega n'est sans doute pas l'unique autel-fétiche de l'espace domestique nankana ${ }^{26}$ qui présente cette caractéristique d'être fixé dans la partie haute d'une chambre ou d'un abri, mais il ressort de certaines indications fournies par Rattray que ce qui pourrait motiver le choix de ce mode d'attache pour le tobega, c'est bien le souci de faire apparaître que, pour tel objet, tout contact avec la terre pourrait avoir des effets funestes. "C'est tres grave ", nous dit Rattray, " si le sac en peau tombe à terre "; il ajoute que c'est pour prévenir ce risque de chute qu'on doit "fermement attacher " le sac en question à la poutre supérieure, à l'intérieur de la case zono. Après le décès du meurtrier, l'obligation de maintenir un écart entre l'autel du meurtre et la terre restera en vigueur pendant un certain temps. Rattray nous indique, en effet, qu'entre le moment où l'on aura été détacher de la poutre où il était fixé le tobega du défunt, et celui où l'on devra le " faire retourner là où il était autrefois ", on sera tenu de le nouer à un bâton planté sur le " tas d'ordure " situé devant la maison. Il restera suspendu à ce bâton pendant trois jours, les trois jours où le corps du défunt sera lui même exposé27.

Comment comprendre l'étrange supplique que présente le spécialiste lors du sacrifice du chien ? Pourquoi, en ce moment du procès rituel, l'instance à laquelle s'adresse le sacrificateur est interpellée avec des mots d'où il ressort que ce qui tient lieu de puissance destinataire,

\footnotetext{
${ }^{26}$ La plupart des autels d'une maison nankana - il y en a un grand nombre - sont fixés au sol. Les autels qui ont vocation à trouver leur place sur les terrasses des habitations ne font pas exception.

27 Lorsqu'il décrit les gestes que fait le spécialiste lors de la mise à mort des volailles qui précède l'installation du tobega, Rattray nous fournit une indication sur une précaution rituelle qui pourrait également s'interpréter comme l'obligation d'empêcher un contact avec le terre. Il nous dit que le sacrificateur * prendra grand soin que le sang ne touche pas l'extrémité noire de la queue de l'écureuil *.
} 
c'est ce qui demeure de la personne qui a été tuée. Ces questions que Rattray n'a pas vraiment abordées, on peut peut-être y apporter un élément de réponse.

Avant la phase d'installation du tobega, tous les gestes qu'avait dû exécuter le spécialiste en direction du meurtrier semblaient bien s'ordonner dans une conduite dont la visée d'ensemble était de défaire cette espèce de ligature qui, depuis l'instant du meurtre, et sous l'effet du rapprochement opéré lors de l'accomplissement de cet acte, avait comme soudé l'un à l'autre deux êtres d'abord distincts. Au terme de ce procès de déliaison, un dernier acte avait lieu qui semblait répondre au souci de reterritorialiser la victime du meurtre. Ce qui fait effet de surprise dans la formule de sacrifice que prononce le spécialiste lors de la fabrication du tobega, c'est l'imploration faite à la victime du meurtre de bien vouloir se fixer dans l'autel, et donc dans une zone de proximité dangereuse par rapport à l'espace de vie du meurtrier. On peut, en effet, s'étonner que ce qui a été dénoué dans les premiers temps du rite, la suite du rituel se donne pour objet de chercher à le renouer.

Comme on l'aura pressenti, il n'y a pas en fait de contradiction entre les deux phases du rituel car le lien que le spécialiste, par son imploration, cherche à établir entre la victime du meurtre et le meurtrier par la médiation d'un autel n'est pas un lien d'attache permanent, mais un lien qui présente toutes les caractéristiques d'un lien cultuel. Or ce qui définit un lien cultuel, c'est qu'il ne s'actualise qu'à des intervalles de temps réguliers et dans un espace strictement délimité. Si contraignant que soit un tel lien dans le cadre du culte que doit rendre le meurtrier auprès de son autel-écureuil, il implique une forme de domestication de la puissance objet du culte et met en jeu un cycle temporel où à des phases de resserrement et de raffermissement font suite des périodes de mise à distance. Le temps de fabrication du tobega marque bien un tournant dans la vie du meurtrier. Le spécialiste par son sacrifice attend de la puissance un signe par laquelle elle dira le choix qu'elle veut faire entre les deux termes de l'alternative qui lui est présentée : soit prendre le chemin de l'errance et laisser le meurtrier " à sa solitude », soit accepter de s'inscrire dans les limites d'un espace cultuel. Cette « manœuvre » du spécialiste fait apparaître sous 
un jour assez sombre le sort qui attend le meurtrier. On ne sait pas très bien ce qu'implique cette menace de solitude qui pèserait sur la vie d'un meurtrier privé de tobega, mais on peut pressentir qu'une telle privation l'exposerait au risque d'un retour intempestif de « l'âme errante " de sa victime. Ce qui, en revanche, nous est connu, c'est le prix qu'aura à payer le meurtrier au cas où la puissance, répondant au souhait du sacrificateur de ne pas être "une mauvaise personne ", accepterait de se lier au meurtrier dans le cadre d'une relation sacrificielle.

\section{Les morsures de l'autel du meurtre}

Ce meurtrier dont Aboya, dans l'exemple analysé, retrace l'itinéraire rituel, on ne sait pas exactement quelle position statutaire il occupait dans sa maison paternelle avant qu'il n'ait commis l'acte qui fit de lui un homme « frappé par le sang ». On sait seulement qu'il était déjà un homme adulte (en âge de mener une action de représailles), mais l'on ignore s'il avait le statut de maître de maison. Dans le cycle ordinaire de la vie d'une maison, le droit de gérer la case rituelle du zono (une case qui joue un rôle très important dans les cycles cérémoniels des Nankanse, en particulier, lors des grandes funérailles) est une prérogative du maître de maison ${ }^{28}$. Bien que Rattray n'ait pas lui-même soulevé cette question, son lecteur, au vu des faits présentés, est amené à se demander ce que pouvait bien devenir cette prérogative du maître de maison une fois que le spécialiste avait mis en place dans le zono un espace cultuel réservé au meurtrier. On peut imaginer deux cas de figures. Dans le cas où le personnage qu'on devait réintégrer dans la maison n'était autre que le maître de maison lui-même, il n'était peut-être pas nécessaire de prévoir un transfert de responsabilité pour la gestion rituelle du zo Dans le cas contraire, on peut supposer que l'attribution au meurtrier

${ }^{28}$ Rattray nous signale que lorsque le maître de maison atteint un âge avancé, c'est dans cette case rituelle qu'il doit se tenir de jour comme de nuit. 
d'un espace réservé dans le zọo pouvait avoir pour effet de déposséder le maitre de maison de la place réelle et symbolique qu'il occupait dans cet espace rituel. Dans l'un ou l'autre cas de figure, l'introduction dans le zono d'un autel de meurtre ne pouvait pas rester sans effet sur la fonction rituelle de cette case. Rattray nous indique en effet que durant toute la période où s'y trouve logé l'autel-écureuil, cette case est interdite d'accès à toute autre personne que le meurtrier. Cette indication met bien en lumiere les effets du tobega sur les structures de l'habitat et les usages de l'espace cérémoniel. En temps ordinaire, un zono est une case qui a pour fonction symbolique d'assurer une communication entre le monde des vivants (les habitants de la maison), d'une part, le monde de leurs ancêtres et de leurs dieux, d'autre part, et c'est bien cette position charnière entre deux mondes qui rend cet espace apte à certains usages rituels au moment des rites de passage. Avec l'introduction de l'autel de meurtre, cette case devenait un sanctuaire secret. On peut donc faire l'hypothèse qu'une pareille transformation devait modifier les conditions de réalisation des rites de passage et, peut-être bien aussi, la façon de penser le rapport aux ancêtres et aux dieux de la maison.

Le culte que le meurtrier devait rendre à son tobega dans l'espace du zono impliquait les obligations suivantes: 1) deux fois par an, il était tenu d'aller nourrir la puissance par des libations de bière de mil et par des versements de sang provenant d'un couple de victimes animales ; 2) durant les mois des récoltes, il devait offrir à cette même puissance les prémices des fruits de la terre en prenant garde de n'oublier aucune des plantes cultivées qui entrent dans l'alimentation de base des Nankanse (premier petit mil, premier maïs, premier sorgho, premières feuilles et premieres fèves de haricots blancs, premières arachides). Avant de parler des immolations d'animaux qui sont périodiquement imposées au meurtrier, essayons de dégager quelques-unes des implications de cette autre contrainte qui lui est faite de ne pouvoir se nourrir des nouveaux fruits de l'année avant de donner au tobega les prémices de chacun de ces produits de la terre.

Rattray décrit assez longuement les rituels qui se succédaient dans une localité quand le temps était venu d'offrir aux dieux et aux ancêtres les prémices. Pour chacun des produits de la terre, le rituel des 
prémices se déroulait en deux phases. Au premier temps, chaque aîné de section clanique, assisté parfois des aînés de lignage, devait se rendre dans le bois sacré où se trouvaient les autels des puissances qui contrôlaient la portion du territoire dont la gestion rituelle lui était confiée. En ce lieu, l'aîné de section, s'adressant aux ancêtres et aux dieux du territoire, faisait ses offrandes en versant soit de l'eau mélangée à la nouvelle plante (telle variété de petit mil, de sorgho, etc.), soit de la bière de nouveau mil. Dans un second temps, les différents maîtres de maison se rendaient dans leurs demeures respectives pour aller offrir les prémices aux dieux et aux puissances présents dans les autels de maison.

Nous ne savons pas si les gens de la maison du meurtrier étaient, ou non, tenus à l'écart de ces rites collectifs, mais on peut supposer que l'installation, avec le tobega, d'une puissance jalouse de ses droits sur les fruits de la terre ne devait pas être sans conséquence sur la façon dont ces gens célébraient les fêtes des prémices. Rattray et Aboya y insistent : aucun des membres de la maison du meurtrier n'avait le droit de consommer ce qu'ils avaient semé ou planté avant que leur parent voué au tobega n'ait donné à cette puissance une offrande des premiers fruits, offrande dont il devait lui-même consommer une part. Un point reste cependant indéterminé. Est-ce que la puissance nouvelle venue dans la maison dépossédait de leur droit aux prémices toutes les autres puissances de la maison, ou bien trouvait-elle sa place et son rang propres parmi celles-ci pour venir à son tour prendre sa part? Consommer avec ses parents un fruit de la terre consacré à un dieu crée entre chacun des commensaux et ce dieu un lien de dépendance durable, et un tel lien de dépendance n'est pas sans risque. Il expose celui ou celle qui manque à ces obligations à la colère du dieu. Des données succinctes rapportées par Rattray sur la question des prémices offertes au tobega, l'on peut au moins tirer cette conclusion minimale.

Une autre remarque d'Aboya (une simple notation) éveille l'attention. Alors qu'il cherche à expliquer ce qui singularise le comportement du meurtrier dans sa façon d'offrir au tobega les prémices, l'informateur de Rattray établit un parallèle entre le détenteur d'un tel autel et une femme qui a accouché des jumeaux, et il justifie cette 
comparaison dans les termes suivants : une mère de jumeaux, nous dit-il, « doit d'abord manger les prémices de tout ce qu'elle a semé avant que quelqu'un d'autre puisse toucher les récoltes " (Rattray, opus cit. : 205, note 1). A la naissance des jumeaux, un spécialiste détenteur d'un autel de type dogele est amené sur les lieux de l'accouchement et installe pour eux une calebasse pleine de racines, calebasse qui, comme le tobega, aura pour caractéristique d'être un autel suspendu. A chaque nouvelle récolte, toute la famille réunie devra en présence des jumeaux offrir à cet autel les premiers fruits de la terre, et ce n'est qu'au terme de cette cérémonie que la famille pourra aller récolter ses champs. Dans un grand nombre de sociétés " voltaïques ", le retour du chiffre deux est une caractéristique des rites associés aux meurtriers (deux autels, deux victimes, deux sacrifices par an, etc.). Parfois, cette obsession du deux est mise en rapport avec le lien de nature gémellaire qui caractérise la relation entre le meurtrier et sa victime. Parfois aussi, elle est corrélée à ce double couple que constituent les jumeaux et leurs géniteurs. S. Dugast, ici même, a bien dégagé ce point a propos des Bassar. La notation d'Aboya sur la mère des jumeaux nous met en présence d'un jeu d'équivalence qu'on pourrait être tenté d'interpréter dans les termes suivants : le meurtrier serait par rapport à la puissance de son autel double ce qu'est une mère de jumeaux par rapport à cette puissance double qu'en engendrant des jumeaux elle a introduite dans son monde familier. Les données ethnographiques sur cette question sont cependant trop restreintes pour qu'on puisse envisager d'approfondir cette comparaison. Toutefois, il nous semble que le rapprochement fait par Aboya est sur un point tout à fait éclairant. Il ressort des données que la puissance gémellaire contenue dans la calebasse des jumeaux est une puissance aux exigences sans limite par rapport à ce qu'elle considère être son « dû " dans les produits sortis des champs des parents de jumeaux, et à l'horizon de cette exigence, il y a toujours menace de ruine. Qu'une menace de ruine pour la maison du meurtrier soit là toujours présente à l'horizon des exigences répétées du tobega, c'est ce que semble vouloir indiquer Aboya lorsque, cherchant à expliquer ce qui rend nécessaire l'acquisition d'un tobega, il rappelle cette sorte de ruine à laquelle le meurtrier, par son acte, a exposé la famille de la victime. 
Chez les Kasena, nous indique ici D. Liberski, l'installation du meurtrier dans son statut de desservant d'un culte sacrificiel rendu à dwaka était censée mettre un terme aux assauts répétés de la victime " demandant le corps du meurtrier". Nous avons nous-même insisté sur le fait qu'à partir du moment où le meurtrier nankana pouvait instaurer avec la puissance du tobega une relation sacrificielle, il n'était plus en proie à de tels assauts. Ceci rappelé, nous allons voir que cette relation sacrificielle n'était pas sans comporter de grands périls pour le meurtrier, la relation en question prenant même parfois une forme si singulière qu'elle exposait le sacrifiant à la morsure mutilante de la puissance à laquelle il rendait un culte.

Deux fois par an, le meurtrier devait entrer dans le zono, non cette fois pour y offrir les premiers fruits de la terre, mais pour y sacrifier un couple d'animaux : chien et chèvre (selon Aboya), chien rouge et volaille (selon Rattray). Sur le déroulement de ces rites, nous n'avons guère d'informations, sauf sur la phase finale, celle où se faisait le partage des viandes sacrificielles. Rattray nous indique que la consommation de cette viande était rigoureusement interdite à tous ceux qui ne faisaient pas partie de l'ensemble des personnes qui, dans le village, avait dû, à la suite d'un meurtre, faire l'acquisition d'un tobega. Cet auteur ajoute que le sacrifiant se devait d'inviter tous ceux qui, dans son entourage, avait ce statut pour leur demander de partager avec lui la nourriture sacrificielle. Rattray n'a pas cherché à approfondir la fonction de ce repas sacrificiel qui réunissait périodiquement tous les détenteurs de tobega autour de parts prélevées sur la dépouille d'un chien. Il n'a pas non plus cherché à savoir si l'ingestion périodique de cette viande sacrificielle n'avait pas pour effet de souder les uns aux autres les individus d'un village qui rendaient un culte à un autel de meurtre, transformant ainsi une population hétérogène en une sorte de « groupe en corps », une confrérie d'homicides « mangeurs de chien ». Enfin, il a laissé en suspens l'énigmatique question du pouvoir inquiétant que les Nankanse, comme d'autres populations voltaïques (S. Dugast et D. Liberski l'ont bien montré), conferent aux viandes des animaux immolés au temps des rites d'enterrement comme au temps des rites liés au meurtre. 
Une brève note d'Aboya nous livre une information supplémentaire sur les sacrifices offerts au tobega, information selon nous tellement importante qu'elle nous amène sinon à réviser complètement, du moins à nuancer l'idée exprimée plus haut qu'un lien sacrificiel noué avec une puissance dans le cadre d'un culte régulier implique une forme de domestication de celle-ci. En fait, dans le cas du tobega, cette domestication n'est jamais assurée, car on ne pourra jamais savoir si pareille puissance saura maintenir dans des limites convenues d'avance ses exigences quant à la nature et au montant du tribut sacrificiel qu'elle réclame au meurtrier. Aboya nous rapporte en effet qu'en certaines situations (il ne précise pas lesquelles), le meurtrier apprend du devin que son tobega réclame « de la chair humaine ». Il ajoute que si le meurtrier n'est pas lui-même un sorcier, il se verra alors contraint de * se couper un doigt ou un orteil " et de « nourrir son tobega avec cela ». Des modèles de sacrifices où la victime sacrificielle tiendrait lieu de substitut de la seule matière oblatoire que convoiteraient les dieux ont fait l'objet de tant de gloses qu'il nous parait inutile de reprendre ici l'examen de cette question. Nous pensons d'ailleurs que l'examen d'une question aussi générale nous éloignerait des questions particulières que soulève l'étrange coutume relevée par Aboya. Ce qu'il faut retenir du discours qu'il a recueilli, c'est ce qui s'y exprime de la crainte fantasmatique qu'inspire la puissance de l'autel du meurtre quant à la forme d'assujettissement où elle tient l'homme qui lui rend un culte. Durant toutes les phases de la cure qui précèdent l'installation du culte, le meurtrier est la proie de sa victime. Durant la période d'exercice de son culte, il se libère de l'emprise de celle-ci pour tomber sous celle d'une puissance qui ne menace plus directement sa vie, mais qui est néanmoins susceptible de le mutiler progressivement.

Au tout début de son propos sur le tobega, Rattray tente de condenser en une formule ce qui lui apparait être le ressort secret de l'obligation faite au meurtrier nankana de rendre un culte sacrificiel à une puissance qui, certes, ne se confond pas avec l'esprit errant de sa victime, mais qui, sous bien des aspects, apparait comme l'instance qui la représente dans la maison du meurtrier. Nous n'avons pas voulu mentionner cette formule de Rattray au début de notre présentation du 
tobega, et ce, de crainte qu'en la citant hors contexte, elle ne retienne guère l'attention du lecteur. Le culte rendu au tobega étant maintenant connu dans ses grandes lignes, nous pouvons faire ressortir ce qui fait l'intérêt de la formule en question. Rattray dit en substance ceci : si le meurtrier doit faire un tobega, c'est qu'il a par son acte privé un esprit de son droit de recevoir des sacrifices coutumiers de son fils. Il ajoute que tout se passe comme si, à travers les sacrifices qu'il allait être amené à faire toute sa vie, le meurtrier ne faisait rien d'autre que de "prendre en charge cet esprit même ", et cela, en lui faisant les offrandes qu'il aurait reçues en des circonstances ordinaires d'un membre de sa propre famille. En première lecture, ces propos nous avaient paru quelque peu arbitraires, car ils semblaient attribuer au camp du meurtrier un souci éthique de réparer un dommage infligé au camp de la victime. Sans écarter à priori l'idée qu'un souci éthique de ce type puisse inspirer le comportement du groupe du meurtrier, il nous apparaissait qu'en la circonstance, un tel principe d'explication était superflu. En fait, nous n'avions pas su discerner que Rattray n'appuyait pas ses propos sur une théorie toute faite d'une obligation morale de réparation pour un dommage fait à l'Autre, mais sur le constat que le meurtre en ces sociétés, en provoquant une " mal mort ", faisait un effet de disruption sur le dispositif sacrificiel, et à travers ce dispositif même, sur le fonctionnement du culte qui, en ce type d'ethnie, permet à chacun des membres d'un lignage de trouver sa juste place dans la généalogie, à savoir ce culte qu'on appelle couramment le culte des ancêtres.

Chez les Nankanse, le fïls aîné devait à la mort de son père reprendre un certain nombre d'autels que ce dernier entretenait de son vivant par le moyen de sacrifices réguliers, mais il devait aussi construire un autel pour son pere mort, et cela, afin de permettre au disparu de s'inscrire dans la chaîne généalogique qui, dans un lignage donné, relie les membres vivants de ce lignage à ses premiers ancêtres fondateurs. La structure de ces cultes lignagers rendus à des ancêtres ou à des esprits de toute nature liés à ces ancêtres est si complexe que nous ne pouvons développer ce point. Il nous suffira d'indiquer que, pour les Nankanse, toute personne du lignage qui a été victime d'un meurtre est appelée à devenir un de ces mauvais morts dont l'esprit est 
condamné à l'errance et qui ne pourra retrouver sa place dans le culte du lignage qu'après une longue période de temps et après l'effectuation de toute une série de rites de "rattrapage ". Rattray nous indique que lorsqu'un tel mauvais mort laisse un fils, ce dernier ne pourra pas constituer d'autel de père. Un tel autel, nous l'avons vu, installe le défunt dans son statut d'ancêtre, mais nous devons ajouter que c'est ce même autel qui assure au fils, par le moyen d'un culte sacrificiel rendu au pere, ce qui va lui permettre d'avoir sa place dans le lignage. Il ressort de ces données que ce qui s'énonce des effets du meurtre dans le discours rituel des Nankanse, ce n'est pas seulement le dommage qu'est la prise d'une vie dans le groupe, mais c'est aussi la brèche qu'il ouvre dans l'édifice symbolique sur lequel repose le lien de filiation et tout l'équilibre du lignage. Certaines données de Rattray montrent qu'aux fils dont le père est mort d'une malemort, le rituel assigne deux types distincts de statut sacrificiel, avec d'un côté, ceux dont les pères sont morts d'une mort étrange (suicidés, varioleux, lépreux, victimes d'une morsure de serpent), de l'autre, les fils de personnes tuées ou assassinées. Le trait distinctif, c'est la possibilité pour ces fils d'avoir ou non un lien sacrificiel très particulier avec un autel - l'autel yini ${ }^{29}$ - qui est associé d'une certaine façon à l'esprit errant du père, mais qui n'est pas un autel de père. Seuls les fils d'un homme victime d'un meurtre sanglant ont droit a cet autel, mais rien n'indique que cette prérogative ait pour effet de conforter leur place dans le lignage. Tout au contraire.

Ces explications une fois données, nous pouvons revenir au tobega. Aux rituels positifs et négatifs qui ont lieu dans la maison de la victime d'un meurtre répondent les rituels effectués dans la maison d'un meurtrier. Chacune des parties prend en compte les effets d'obligation rituelle que la puissance libérée par le meurtre produit chez l'Autre. Il n'y a pas besoin d'un arbitrage entre la famille du meurtrier et la famille de la victime. Certes, l'installation du tobega requiert l'intervention d'un spécialiste, mais ce dernier n'agit pas en position

${ }^{29}$ Très étrange autel placé sur les terrasses des maisons, et qui semble lié à ces autels du destin, appelés du même nom, auxquels rendent un culte un très grand nombre d'individus. 
de tiers, comme pourrait le faire le maître de la Terre dans une situation de meurtre où les deux parties seraient tenues de venir " ramasser le sang ". Malgre l'absence d'une pareille instance tierce, on peut tout à fait concevoir (c'est ce qu'a fait Rattray) que le meurtrier n'ignore pas que la puissance qu'il nourrit par ses sacrifices sur l'autel du tobega, c'est une puissance de père laissée pour ainsi dire en déshérence, une puissance qui dès lors qu'elle est privée des sacrifices qu'elle aurait dû recevoir dans la maison du fils désertera celle-ci pour devenir puissance sacrificielle dans la maison du meurtrier. Les actes sacrificiels du meurtrier apparaissent donc comme une sorte de réponse en miroir de la situation sacrificielle faite au fils de sa victime. Rattray ne donne pas d'indications sur les conséquences que peuvent avoir sur le lien à l'autel du père l'acquisition pour le fils d'un statut rituel de meurtrier, mais on voit mal comment ce dernier pourrait tout à la fois prendre en charge un autel de père qui le rattache à sa lignée et un autel de meurtre qui le met en connection avec la lignée de sa victime.

Nous avons déjà donné une première idée de ce que l'on fait de l'autel du meurtre après la mort de son détenteur, mais nous n'avons pas encore indiqué que c'est le fils aîné du meurtrier qui devra prendre l'initiative de l'enlèvement et de la mise à l'écart de cet autel. Selon Aboya, la démarche du fils aîné consistera à réunir plusieurs meurtriers de son village détenteurs de tobega (sans doute les mêmes personnes que celles avec lesquelles le défunt, de son vivant, partageait les viandes sacrificielles) pour fixer avec eux le moment du rite d'enlevement. Nous savons déjà qu'une fois l'autel enlevé, il faudra l'éloigner de la maison du meurtrier. Pour restituer la façon dont se fera cette mise à l'écart, il n'y a pas de meilleurs mots que ceux d'Aboya : " Ils partent en direction du village dont était originaire la personne tuée ou assassinée. Quand ils atteignent la vallée, ils jettent l'autel au loin afin qu'il (l'esprit de l'homme tué) puisse partir parce que son propriétaire n'est désormais plus en vie et qu'il doit retourner chez lui... " (Rattray, 1932 : 203).

Avec ce dernier acte, on a bien l'impression que le cycle du meurtre sera pour ainsi dire bouclé, et qu'avec ce mouvement de retour de la puissance au lieu où elle s'origine, ce qui a été arrêté dans le cycle de la vie rituelle des maisons des deux parties pourra 
reprendre son cours normal. L'examen des coutumes nankanse qui s'articulent autour de la notion de segere, " esprit gardien ${ }^{30}$, nous montre qu'il n'en est rien. A la lecture des faits que rapporte Rattray sur des procès d'adoption d'enfants par des esprits gardiens, on s'aperçoit qu'il n'est pas rare que le devin consulté pour des histoires de naissance soit amené à dire aux consultants que ce qui arrive à l'enfant - l'enfant à naître ou l'enfant déjà né - c'est un signe qu'a émis un tobega pour faire savoir qu'il voulait devenir l'esprit gardien de cet enfant. Auprès de la chambre d'un tel enfant, on posera une poterie emplie de racines, sorte d'autel, que le pupille ainsi adopté devra entretenir toute sa vie par des sacrifices. Tout se passe donc comme si, durant la vie du meurtrier, la puissance du tobega ne s'était pas résignée à se fixer dans les limites de l'espace cultuel où l'on avait voulu l'enfermer, mais qu'elle s'était répandue en divers lieux pour venir enfin s'agglutiner à des enfants. Quels enfants? Rattray nous apprend qu'Aboya, son principal informateur pour son enquête ethnographique autour de la question du meurtre, avait lui-même été directement impliqué dans une affaire de tobega devenu esprit gardien d'un enfant. L'enfant en question était le propre enfant d'Aboya, celui qu'il avait eu d'une femme qui, quelques années auparavant, avait frappé à mort sa co-épouse. A l'époque où l'épouse meurtrière mit au monde l'enfant d'Aboya, l'esprit tobega qui provenait de la femme assassinée fit savoir au devin qu'il voulait devenir l'esprit gardien de cet enfant ${ }^{31}$. Rattray ne commente pas d'avantage cette fantastique histoire, et ne nous dit pas les conséquences qui en résultèrent pour l'enfant, un enfant qui allait devoir vivre sous la garde de l'esprit d'une mère classificatoire, non pas l'esprit de n'importe quelle " mère » défunte, mais celui d'une « mere " autrefois frappée à mort par sa propre mère. Mais dans l'ouvrage de Rattray sont mentionnés encore bien d'autres

\footnotetext{
${ }^{30}$ Selon Rattray, il n'y a pas que les esprits ancestraux (en lignée paternelle et maternelle) qui puissent remplir la fonction d'esprit gardien d'un enfant à naître. Toute une série de puissances ou de choses peuvent avoir également cette fonction.

${ }^{31}$ Est-ce à dire qu'une femme qui avait tué pouvait avoir en cette société un statut rituel de meurtrier, et donc entrer en possession d'un autel tobega ? Rattray ne précise pas ce point.
} 
situations qui mettent en cause des histoires d'adoption d'enfant par un esprit gardien provenant d'un tobega. Une note d'Aboya extraite d'un de ses compte rendus sur les rites funéraires d'une vieille femme morte dans la maison de son mari met en pleine lumière l'amplitude du champ où la puissance du tobega se propage par sa capacité à s'agglutiner aux enfants dont elle veut devenir l'esprit gardien. Lors d'une séquence des rites funéraires, l'on doit « balayer » le corps du défunt - de tout défunt - pour le débarrasser de ce qu'il a gardé des imprégnations de son esprit gardien. Dans son compte rendu, Aboya note ce qui fut dit et fait à cette étape du balayage lors des funérailles de la vieille femme. La famille du mari, nous dit-il, envoya des messagers dans le village natal de la vieille femme pour prévenir ses parents paternels. Avant de mettre le corps en terre, ajoute-t-il, on ne peut se soustraire à cette obligation, car on ne peut jamais exclure que l'esprit gardien d'une personne dont la première histoire de vie est mal connue ne soit un tobega.

Michel Cartry

E.P.H.E. 


\section{Références bibliographiques}

Benvéniste, E.

1969 Le vocabulaire des institutions indo-européennes, vol. 2 , Paris, Editions de Minuit, $340 \mathrm{p}$.

Bonnafé, $\mathrm{P}$. et Fiéloux, $\mathrm{M}$.

1984 * Le dédain de la mort et la force du cadavre. Souillure et purification d'un meurtrier lobi (Burkina:Haute-Volta) ", Etudes rurales, XVC: $63-87$.

Bohannan, P. (edit.).

1960 African Homicide and Suicide. Princeton, Princeton University Press, $270 \mathrm{p}$.

Bohannan, P.

1960 "Homicide among the Tiv of central Nigeria ", in African Homicide and Suicide, pp 30-64.

Cros, $\mathrm{M}$.

1987 Anthropologie du sang chez les Lobi (Burkina Faso, Côte d'Ivoire). Mise en scène d'un tabou, thèse de doctorat, Université de Paris V, 741 p.

Dugast, $\mathrm{S}$.

1992 Rites et organisation sociale. L'agglomération de Bassar au NordTogo, thèse de doctorat, Ecole des Hautes Etudes en Sciences Sociales, $966 \mathrm{p}$.

Evans-Pritchard, E.E.

1940 The Nuer: A Description of the Modes of Livelihood and Political Institutions of a Nilotic People, Oxford, Clarendon Press.

1956 Nuer Religion, Oxford, Clarendon Press, 336 p.

Fortes, M.

1945 The Dynamics of Clanship Among the Tallensi, London, Oxford University Press.

1949 The Web of Kinship Among the Tallensi, London, Oxford University Press.

Frazer, J. G.

1981 Le rameau d'or. Tabou et les périls de l'âme, Paris, Robert Laffont, $1004 \mathrm{p}$.

Guibal, $M$.

1984 "L'étranger. Crise-représentation. *, vol. 2, Actes de la réplique, édité par le collectif Evénements Psychanalyse, Lyon. 
Goody, J.

1962 Death, Property and the Ancestors: A Study of the Mortuary Customs of the LoDagaa of West Africa, Stanford University Press, 452 p.

1967 The Social Organization of the LoWili, Oxford University Press (International African Institute), $123 \mathrm{p}$.

Hegel, G.W.F.

1976 Système de la vie éthique, trad. française, Paris, Payot, $211 \mathrm{p}$.

Junod, Henri-Alexandre

1912-1913

The Life of a South African Tribe, 2 vol., London.

1936 Moeurs et coutumes des Bantous: la vie d'une tribu sud-africaine. Paris, Payot.

Labouret, $\mathrm{H}$.

1916 * La guerre dans ses rapports avec les croyances religieuses chez les populations du cercle de Gaoua (Haut-Sénégal et Niger) *. Annuaire et Mémoires du Comité d'Etudes Historiques et Scientifiques de l'Afrique Occidentale Française, Dakar, pp 289-304.

$1917 a$ * La terre dans ses rapports avec les croyances religieuses chez les populations du cercle de Gaoua (Haut-Sénégal et Niger) ». Annuaire et Mémoires du Comité d'Etudes Historiques et Scientifiques de l'Afrique Occidentale Française, Dakar, pp 305-316.

$1917 b$ * La chasse et la pêche. Dans leurs rapports avec les croyances religieuses parmi les populations du Lobi *. Annuaire et Mémoires du Comité d'Etudes Historiques et Scientifiques de l'Afrique Occidentale Française, Dakar, pp 244-276.

1931 Les tribus du Rameau Lobi. Paris, Institut d'Ethnologie (Travaux et mémoires de l'Institut d'Ethnologie, XV), $510 \mathrm{p}$.

1958 Nouvelles notes sur les tribus du rameau Lobi. Mémoires de L'Institut Français d'Afrique Noire, Dakar, N 54.

Liberski, D.

1991 Les dieux du territoire. Unité et morcellement de l'espace en pays kasena (Burkina Faso), thèse de doctorat, Ecole Pratique des Hautes Etudes, $V^{e}$ section, Paris, 384 p.

Mauss, $M$.

$1969 a$ * Essai sur la nature et la fonction du sacrifice *, in M. Mauss, Oeuvres, vol. 1, pp. 193-354 (d'abord paru dans l'Année Sociologique, 1899, 2).

$1969 b$ * La religion et les origines du droit pénal d'après un livre récent * in M. Mauss, Oeuvres, vol 2, pp. 651-698 (d'abord paru dans Revue de l'Histoire des Religions, 1896, 34). 
Père, $M$.

1988 Les Lobi. Tradition et changement, 2 tomes, Siloë, Laval.

Rattray, R.S.

1932 The Tribes of the Ashanti Hinterland, 2 vol., Oxford, Clarendon Press, 604 p.

Roscoe, J.

1915 The Northern Bantu: An Account of Some Central African Tribes of the Uganda Protectorate, Frank Cassa and Company.

1924 The Bagesu, Cambridge University Press.

de Rouville, C.

1987 Organisation sociale des Lobi (Burkina Faso, Côte d'Ivoire), Paris, L'Harmattan, 259 p.

Steinmetz, M. R.

1894 Ethnologische Studien zur ersten Entwickelung der Strafe, Leiden, O. Harrassowitz, vol. 1.

Traoré, D.

1935 * Notes sur les mœurs et coutumes Lobi », L'Education africaine, $24^{\mathrm{e}}$ année, janvier-mars, $\mathrm{N} 89$, pp 49-57. 